
\title{
R Research Soure \\ Spectrally Efficient IR-UWB Pulse Designs Based on Linear Combinations of Gaussian Derivatives
}

Haidar Taki ( $\nabla$ haidar.taki@lau.edu.Ib )

Lebanese American University https://orcid.org/0000-0002-1288-8874

Chadi Abou-Rjeily

Lebanese American University

\section{Research Article}

Keywords: Ultra WideBand (UWB), Impulse Radio (IR), Federal Communication Commission (FCC), Power Spectral Density (PSD), Global Optimization, Power Efficiency, Linear Combinations, Gaussian Derivatives, Bit Error Rate (BER).

Posted Date: January 17th, 2022

DOI: https://doi.org/10.21203/rs.3.rs-1160492/v1

License: (9) This work is licensed under a Creative Commons Attribution 4.0 International License. Read Full License 


\title{
Spectrally Efficient IR-UWB Pulse Designs Based on Linear Combinations of Gaussian Derivatives
}

\author{
Haidar Taki ${ }^{1^{*}}$ and Chadi Abou-Rjeily ${ }^{1}$ \\ ${ }^{1}$ School of Engineering, Lebanese American University (LAU), \\ Byblos, Lebanon.
}
*Corresponding author(s). E-mail(s): haidar.taki@lau.edu.lb; Contributing authors: chadi.abourjeily@lau.edu.lb;

\begin{abstract}
Pulse design is critical for impulse radio communications, as it determines the transmission efficiency with respect to regulation spectral limits. In this paper, we propose the design of several novel pulse shapes relying on combinations of Gaussian derivatives with the target of improving the spectral efficiency. A general model for maximizing the efficiency of dual and triplet couplings is presented, employing the interior point global optimization algorithm. Then, the spectrum peak frequency is derived in closed form for any combination of two Gaussians with consecutive orders of derivation. The parameters controlling the time properties of the generated waveforms have been adequately adjusted to reach the best compliance with the spectral mask. Novel pulses have been investigated providing a high efficiency using simple generation mechanisms, which can be practically implemented via analog circuits. Results demonstrated the advantage of the proposed pulse shapes in terms of the bit error rate performance for 2 Gbps OOK and 1 Gbps PPM in AWGN and multipath channels.
\end{abstract}

Keywords: Ultra WideBand (UWB), Impulse Radio (IR), Federal

Communication Commission (FCC), Power Spectral Density (PSD), Global Optimization, Power Efficiency, Linear Combinations, Gaussian Derivatives, Bit Error Rate (BER). 


\section{Introduction}

An emerging wireless technology for short-range communications is the Ultra Wide Band (UWB) technique, which is gaining popularity due to its attractive features, including low power consumption, high data rate, robustness to multipath fading in dispersive channels, fine positioning characteristics, and information security [1-3]. UWB has been an appropriate solution in diverse applications, like radar, local and wide area networks, remote sensing, home automation, emergency communications, medical imaging, and tracking [4-11]. Following the regulation of the Federal Communication Commission (FCC) approved in 2002, UWB devices are authorized to operate over the [3.1-10.6] $\mathrm{GHz}$ frequency band within a power limit of $-41.3 \mathrm{dBm} / \mathrm{MHz}$, to maintain a safe coexistence with other radio services [12-14]. A particular UWB approach is the Impulse Radio (IR) baseband scheme, which relies on transmitting data symbols via repetitive pulse signaling, and is characterized by an appealing low-cost transceiver architecture [15-20].

Due to the stringent power and spectral constraints of regulatory bodies, a highly efficient IR transmission is needed, while the utilized pulses play an essential role in determining the power efficiency of the modulated signal [21]. The complexity of the pulse generation technique is a major concern in IRUWB systems, as it indicates their practicality in real-time applications. As illustrated in figure (1), the order of complexity depends on the fabrication technology, pulse derivation order, number of pulses used in combination-based waveforms, devices and components adopted in the configuration, besides to the generation technique, which could be analog, digital, or hybrid. The pulse shaping technology could be electrical, optical, or opto-electrical, while the application scenario determines the candidate technique [21-23]. Optical-based schemes are more complex than electrical alternatives, but mainly suit overfiber networks $[21,24,25]$. The electrical solution is less expensive and better fits wireless transmission systems. In particular, the Complementary Metal Oxide Semiconductor (CMOS) technology has been widely used in literature, as a low-cost simple approach to generate nanosecond pulses at milliwatt power with small chip size $[22,23,26]$. The derivation order actually indicates the number of time-differentiation stages threw which the created pulse has to pass before reaching the target waveform. The combination size determines the number of parallel branches integrated in the generation circuit. Hence, both parameters have an impact on the configuration complexity, as arithmetic operations are mainly performed using analog electronic circuit elements $[27,28]$.

Digitizing the generation system provides a very high flexibility in the pulse design, however, it is the most expensive approach, especially when dealing with GHz-signals. Incorporating a local oscillator to shift the spectrum towards the desired band, significantly increases the level of complicacy, and hence, pure impulse radio schemes have been considered as a good choice for simple and cheap UWB transceivers $[16,18,23]$. In this study, we investigate the design of carrier-free UWB generation using a few number of elementary pulses, so as to 


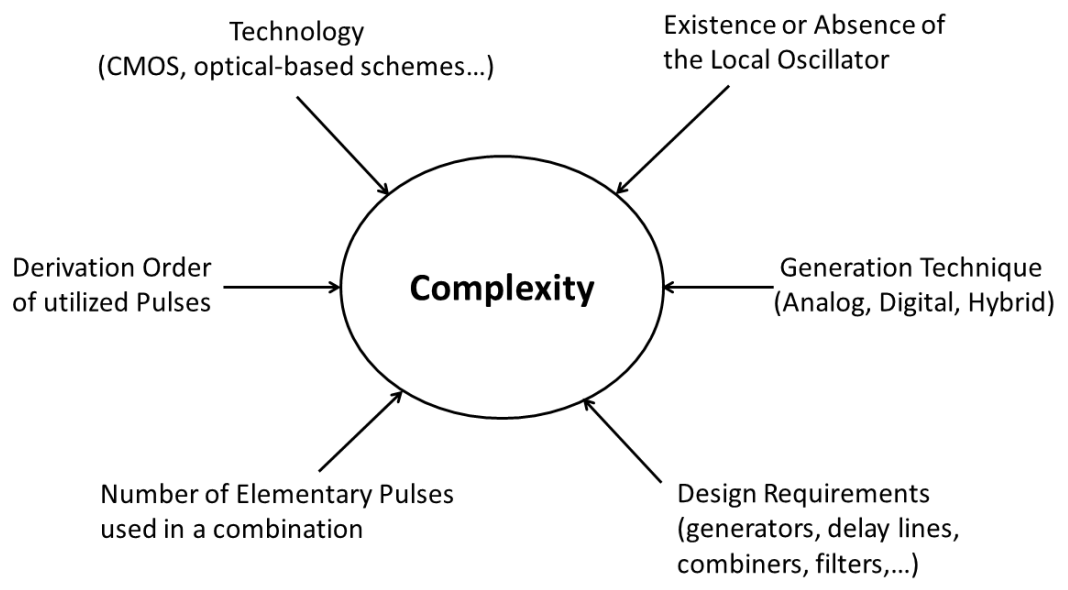

Fig. 1 Determinants of generation complexity for any pulse shaping technique.

synthesize the transmitted signal at acceptable order of complexity and fit the low-cost IR systems demand. Next section summarizes the research progress on pulse shaping, while the contributions of our work are stated in the sequel.

\section{Literature Review}

Various pulse types can be adopted with IR-UWB, mainly the Rectangular, Laplacian, Hermite, Cubic, and Gaussian pulses [15, 29-33]. The latter will be considered in our work due to their good compliance with FCC standard and ease of generation using analog circuits [22, 26, 30, 32, 34-36]. Among the Gaussian family, the $5^{\text {th }}$ derivative pulse is the most conventional waveform which has been widely used in literature [32, 37-42].

Tables (1) and (2) summarize certain previous studies addressing pulse shaping schemes, while the power efficiency has been defined in $[37,38]$ as clarified in the next section. In [43], a linear combination of two Gaussian monocycles with different spreading factors was demonstrated. In [37], a novel waveform is composed from two modified Gaussian doublets, which are identical with reversed polarities, a high efficiency has been acquired using a delay line. In [38], we applied a triplet combination of fundamental Gaussians with different shaping factors, as a pre-distortion approach against nonlinearities inherent to optical devices, and a good efficiency has been recorded. In [24], FCC-compliant UWB pulse is generated based on modified Gaussian quadruplet and incoherent wavelength-to-time conversion. The quadruplet is formed by subtracting a broad Gaussian pulse from two narrow ones having same width and amplitude. Gaussian and programmable filters have been used to shape the spectrum, while the resultant pulse has a high fractional bandwidth and fine power efficiency [24]. In [21], a photonic generation of Gaussianbased pulses has been demonstrated, utilizing dual-polarization quadrature 


\begin{tabular}{|c|c|c|c|c|c|c|c|}
\hline Ref & $\begin{array}{l}\text { Main Principle for } \\
\text { UWB } \quad \text { Waveform } \\
\text { Generation }\end{array}$ & $\begin{array}{l}\text { Num } \\
\text { of } \\
\text { Puls }\end{array}$ & $\begin{array}{l}\text { Max } \\
\text { Derv }\end{array}$ & $\begin{array}{l}\text { Design } \\
\text { Require- } \\
\text { ments }\end{array}$ & $\begin{array}{l}\text { Data } \\
\text { Rate }\end{array}$ & $\begin{array}{l}\text { Power } \\
\text { Effi- } \\
\text { ciency }\end{array}$ & $\begin{array}{l}\text { Additional } \\
\text { Information }\end{array}$ \\
\hline$[43]$ & $\begin{array}{l}\text { Adding Gaussian } \\
\text { monocycles with differ- } \\
\text { ent polarities and pulse } \\
\text { shaping factors }\end{array}$ & 2 & 1 & $\begin{array}{l}\text { Two PGs } \\
\text { and one } \\
\text { adder }\end{array}$ & $\begin{array}{l}2 \\
\text { Gps }\end{array}$ & $48.5 \%$ & $\begin{array}{l}f_{\text {peak }}=6 \mathrm{GHz}, \\
B_{10}=7.5 \mathrm{GHz}, \\
\text { fractional band- } \\
\text { width, } 1.25\end{array}$ \\
\hline [37] & $\begin{array}{l}\text { Adding modified Gaus- } \\
\text { sian doublets with dif- } \\
\text { ferent polarities and } \\
\text { same pulse shaping fac- } \\
\text { tor }\end{array}$ & 2 & 2 & $\begin{array}{l}\text { Two PGs, } \\
\text { one adder, } \\
\text { and one } \\
\text { microwave } \\
\text { delay line }\end{array}$ & $\begin{array}{l}2 \\
\text { Gps }\end{array}$ & $57.4 \%$ & $\begin{array}{l}f_{\text {peak }}=\text { fractional } \\
\text { GHz, fral } \\
\text { bandwidth, } 1.08, \\
\text { modified Gaus- } \\
\text { sian used (not } \\
\text { original) }\end{array}$ \\
\hline [38] & $\begin{array}{l}\text { Linearly combining } \\
\text { fundamental Gaussians }\end{array}$ & 3 & 0 & $\begin{array}{l}\text { Three PGs } \\
\text { and one } \\
\text { adder }\end{array}$ & $\begin{array}{l}2 \\
\text { Gps }\end{array}$ & $\begin{array}{l}56.47 \\
\%\end{array}$ & $\begin{array}{l}\text { Pulses have dif- } \\
\text { ferent polarities }\end{array}$ \\
\hline [24] & $\begin{array}{l}\text { Subtracting a broad } \\
\text { Gaussian } \\
\text { from two narrow } \\
\text { identical } \\
\text { pulses, besides to } \\
\text { applying incoherent } \\
\text { wavelength-to-time } \\
\text { conversion }\end{array}$ & 3 & 0 & $\begin{array}{l}\text { Two } \\
\text { PGs, one } \\
\text { adder, two } \\
\text { microwave } \\
\text { delay } \\
\text { lines, and } \\
\text { optical } \\
\text { devices }\end{array}$ & $\begin{array}{l}2.5 \\
\text { Gps }\end{array}$ & $51 \%$ &  \\
\hline [21] & $\begin{array}{l}\text { Dual-polarization } \\
\text { quadrature phase } \\
\text { shift keying and bal- } \\
\text { anced photo-detection, } \\
\text { applied on a Gaussian } \\
\text { pulse to produce a } \\
\text { quadruplet signal }\end{array}$ & $\begin{array}{l}1 \\
\text { pulse } \\
\text { split- } \\
\text { ted } \\
\text { into } \\
4\end{array}$ & 0 & $\begin{array}{l}\text { One PG, } \\
\text { electrical } \\
\text { and opti- } \\
\text { cal delay } \\
\text { lines, } \\
\text { and four } \\
\text { MZMs }\end{array}$ & $\begin{array}{l}312.5 \\
\text { Mbps }\end{array}$ & $\begin{array}{l}53.46 \\
\%\end{array}$ & $\begin{array}{l}B_{10}=6.88 \mathrm{GHz}, \\
\text { two photo- } \\
\text { detectors are } \\
\text { used in a } \\
\text { balanced mode }\end{array}$ \\
\hline
\end{tabular}

Table 1 Overview for certain studies investigating IR-UWB shaping techniques based on combinations of waveforms (while the number of pulses used is marked as 'Num of Puls'), 'PG' denotes the Pulse Generator, 'Max Derv' actually indicates the maximum order of derivation for the original pulses initially used (not the resultant ones), $f_{\text {peak }}$ is the peak frequency, and $B_{10}$ is the $10-\mathrm{dB}$ bandwidth.

phase shift keying (DP-QPSK). A Gaussian signal has been used to drive four Mach-Zehnder Modulators (MZMs) forming the DP-QPSK system, and an 


\begin{tabular}{|c|c|c|c|c|c|c|c|}
\hline Ref & $\begin{array}{l}\text { Main Principle for } \\
\text { UWB } \quad \text { Waveform } \\
\text { Generation }\end{array}$ & $\begin{array}{l}\text { Num } \\
\text { of } \\
\text { Puls }\end{array}$ & $\begin{array}{l}\text { Max } \\
\text { Derv }\end{array}$ & $\begin{array}{l}\text { Design } \\
\text { Require- } \\
\text { ments }\end{array}$ & $\begin{array}{l}\text { Data } \\
\text { Rate }\end{array}$ & $\begin{array}{l}\text { Power } \\
\text { Effi- } \\
\text { ciency }\end{array}$ & $\begin{array}{l}\text { Additional } \\
\text { Information }\end{array}$ \\
\hline [25] & $\begin{array}{l}\text { Linearly combining } \\
\text { four Gaussian pulses } \\
\text { with the same spread- } \\
\text { ing factor, while } \\
\text { exploiting the ben- } \\
\text { efits of FBG and } \\
\text { applying incoherent } \\
\text { wavelength-to-time } \\
\text { conversion }\end{array}$ & 4 & 0 & $\begin{array}{l}\text { One PG, } \\
\text { spectral } \\
\text { shaper, } \\
\text { three } \\
\text { delay func- } \\
\text { tions, and } \\
\text { optical } \\
\text { devices }\end{array}$ & $\begin{array}{l}2.5 \\
\text { Gps }\end{array}$ & $57.2 \%$ & $\begin{array}{l}\text { Central fre- } \\
\text { quency, } \quad 7.5 \\
\mathrm{GHz}, \quad B_{10}= \\
6.25 \quad \mathrm{GHz}, \\
\text { fractional } \\
\text { bandwidth, } \\
0.83\end{array}$ \\
\hline$[44]$ & $\begin{array}{l}\text { Linearly } \\
\text { different orders of } \\
\text { Gaussian derivatives } \\
\text { which are influenced } \\
\text { by antenna effects }\end{array}$ & 8 & 4 & $\begin{array}{l}\text { Eight PGs, } \\
\text { one adder, } \\
\text { and seven } \\
\text { microwave } \\
\text { delay lines }\end{array}$ & $\begin{array}{l}1.66 \\
\text { Gps }\end{array}$ & $71 \%$ & $\begin{array}{l}\text { Pulses used } \\
\text { (with different } \\
\text { polarities): } 2 \\
\text { monocycles, } 5 \\
\text { doublets, and } \\
1 \text { fourth-order } \\
\text { derivative }\end{array}$ \\
\hline$[46]$ & $\begin{array}{l}\text { Applying a series of } \\
\text { Gaussian functions } \\
\text { with an incrementally } \\
\text { increasing order of } \\
\text { derivation }\end{array}$ & 15 & 15 & $\begin{array}{l}\text { Fifteen } \\
\text { PGs and } \\
\text { one adder }\end{array}$ & $\begin{array}{l}55.55 \\
\text { Mbps }\end{array}$ & $\begin{array}{l}\leq 50 \% \\
\text { Appar- } \\
\text { ently }\end{array}$ & $\begin{array}{l}\text { Large num- } \\
\text { ber of pulses } \\
\text { used and high } \\
\text { derivation } \\
\text { order }\end{array}$ \\
\hline$[47]$ & $\begin{array}{l}\text { Shaping the generated } \\
\text { waveforms via FIR } \\
\text { band pass filtering }\end{array}$ & $\begin{array}{l}\text { Likely } \\
37\end{array}$ & 0 & $\begin{array}{ll}\text { One } & \text { PG } \\
\text { and } & \text { FIR } \\
\text { filter } & \end{array}$ & $\begin{array}{l}\text { Not } \\
\text { men- } \\
\text { tioned }\end{array}$ & $91.2 \%$ & $\begin{array}{l}B_{10} \approx 7.5 \\
\mathrm{GHz}, \quad \text { high- } \\
\text { length filter } \\
\text { (complex } \\
\text { design) }\end{array}$ \\
\hline
\end{tabular}

Table 2 Overview for certain studies investigating IR-UWB shaping techniques based on combinations of waveforms (while the number of pulses used is marked as 'Num of Puls'), 'PG' denotes the Pulse Generator, 'Max Derv' actually indicates the maximum order of derivation for the original pulses initially used (not the resultant ones), $f_{\text {peak }}$ is the peak frequency, and $B_{10}$ is the $10-\mathrm{dB}$ bandwidth.

efficient UWB quadruplet has been produced via balanced photo-detection [21]. Spectral efficient pulses have been designed in [25] using polarizationmaintaining Fiber Bragg Grating (FBG) and incoherent wavelength-to-time mapping. In [44], a Gaussian monocycle and its higher-order derivatives have been combined to build new waveforms taking into account antenna effects, where improvements in terms of the spectral efficiency were achieved compared to [45]. In [46], a series of 15 Gaussian functions has been applied, while the order of derivation has been increased from 1 to 15 among the series terms, and 
the elementary pulses' parameters have been optimized using the Firefly algorithm. A high efficiency was recorded in [47], using a bandpass finite impulse response (FIR) filter which has been numerically implemented via digital techniques. The spectral behavior of the Gaussian pulses proposed in [28] and [5] can be further improved using a simultaneous combination of pulses. In [48], the authors investigate the design of a set of pulses that satisfy orthogonality property, relying on the eigenfunctions of Sturm-Liouville boundary value problems.

As reported in the previously delineated studies, the performance of single waveforms is limited by the number of variables involved, hence, applying a combination of pulses is a pertinent solution for efficiently designing the IRUWB spectra and collecting the maximum possible energy without violating FCC mask. In this contribution, four new pulse shapes have been developed, $4^{t h}-5^{t h}$ Gaussian couple, dual $4^{\text {th }}$ Gaussian combination, $5^{t h}-6^{t h}$ Gaussian couple, and $6^{\text {th }}-7^{t h}-7^{\text {th }}$ Gaussian triplet. The proposed UWB waveforms allow to achieve different levels of trade-off between power efficiency and complexity. In section 3, a simple optimization model using the interior point algorithm is described [49-52], based on which we optimize the targeted pulse combinations. In section 4, the peak frequency expression has been derived for a general form of two combined Gaussians with consecutive orders of derivation. In section 5, we detail the novel combinations proposed and measure their power spectral efficiency with respect to FCC mask. In section 6, a performance evaluation for the identified waveforms is illustrated in terms of error probability, while the obtained results are promising for future impulse radio applications.

\section{General Approach to Design UWB Pulses Based on Combinations of Gaussians}

The time domain formula of the fundamental Gaussian pulse is expressed as $[32]$ :

$$
p_{\sigma, 0}(t)=\frac{A}{\sqrt{2 \pi \sigma^{2}}} e^{-\frac{t^{2}}{2 \sigma^{2}}}
$$

where $A$ is the amplitude and $\sigma$ is the pulse shaping factor or spreading factor. The $k^{t h}$ derivative Gaussian can be recursively obtained as:

$$
p_{\sigma, k}(t)=-\frac{k-1}{\sigma^{2}} p_{\sigma, k-2}(t)-\frac{t}{\sigma^{2}} p_{\sigma, k-1}(t)
$$

Our aim in this study is to design novel pulse shapes providing efficient impulse radio transmission based on combinations of Gaussian pulse derivatives. In order to achieve this goal, the parameters involved in the pulse generation technique have to be adequately adjusted to optimize the spectrum of the resultant waveform. A linear combination of delayed Gaussians has the following general 


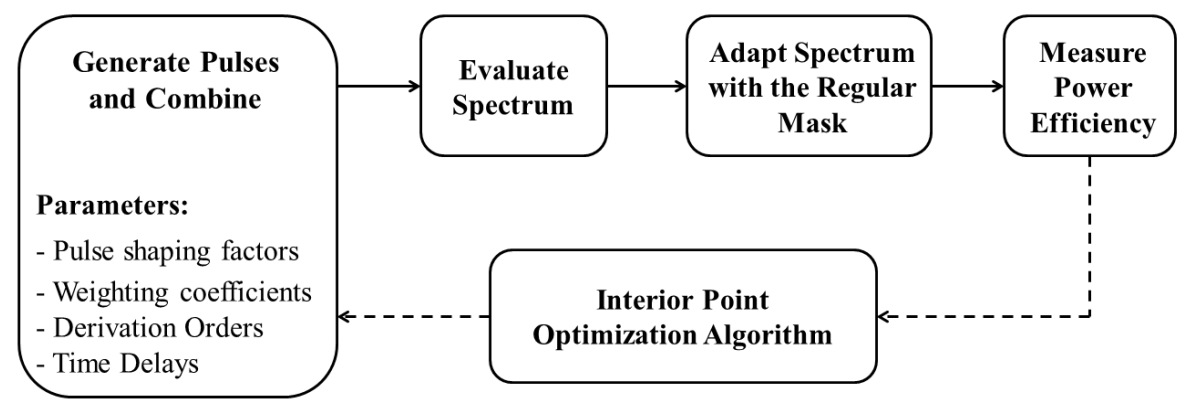

Fig. 2 A schematic for the optimization mechanism used to create UWB pulse combinations.

expression:

$$
c(t)=\sum_{i=1}^{N} a_{i} p_{\sigma_{i}, k_{i}}\left(t-\delta_{i}\right)
$$

where $a_{i}$ is the weighting coefficient and $\delta_{i}$ is the time delay of basis pulses. $N$ is the number of waveforms used, which has been preferably selected not to exceed 3 in order to avoid adding too much complexity to the generation system, and keep on simple configurations involving few parallel circuits based on analog devices [22]. To evaluate the spectrum with respect to FCC mask, we address the power efficiency regarded in [38] which has been expressed as:

$$
\eta=\frac{\int_{3.1 G H z}^{10.6 \mathrm{GHz}} S(f) \mathrm{df}}{\int_{3.1 G H z}^{10.6 G H z} S_{F C C}(f) \mathrm{df}} \times 100 \%
$$

where $S(f)$ denotes the power spectral density of the generated waveform and $S_{F C C}(f)$ stands for the FCC mask [37]. As it is difficult to reach an analytical solution for the integral in equation (4), we adopt a numerical approach to evaluate the power efficiency. Equation (3) informs that the parameters to be optimized are mainly the pulse shaping factors, derivation orders, weighting coefficients, and time delays. In such a multi-variable problem, a global optimization scheme is recommended, where the interior point method has been the candidate algorithm [49-52]. Figure (2) describes the optimization procedure, the arbitrary parameters of utilized pulses are iteratively tuned to realize the maximum power efficiency of their adapted spectrum, the latter denotes the obtainable spectrum after being adjusted in amplitude to fit the radiation mask. This sequential system has been completely implemented in MATLAB. Relying on this model, we explore several pulse combinations which seem to be pertinent for impulse radio signaling, as contributed in the sequel. In the next section, we highlight on the peak frequency, as an important criterion which determines the spectral behavior in correspondence with the emission mask. 


\section{Peak Frequency And Spectral Compliance with the Radiation Limit}

The spectral characteristics of any UWB waveform have to be considered so as to evaluate the spectrum in view of emission standards, and one of the key terms is the peak frequency $f_{\text {peak }}$. Carrier-based schemes provide simplicity in centering the spectrum on any target band by just tuning the frequency of local oscillator. Conversely, the spectrum location of impulse radio depends on the time properties determined by the pulse/pulses parameters. For the $k^{t h}$ Gaussian, the peak frequency of the spectrum is expressed as [32]:

$$
f_{\text {peak }}=\frac{\sqrt{k}}{2 \pi \sigma}
$$

With combinations of Gaussians, $f_{\text {peak }}$ depends on more variables, where for complicated combinations the peak frequency can not be derived in closedform.

Consider a simple combination of Gaussian pulses having consecutive orders of derivation ( $k$ and $k+1$ ). The spectra of proposed pulses can be derived as [32]:

$$
\begin{gathered}
P_{k}(f)=A_{1}(2 \pi f j)^{k} e^{-2(\pi f \sigma)^{2}} \\
P_{k+1}(f)=A_{2}(2 \pi f j)^{k+1} e^{-2(\pi f \sigma)^{2}}
\end{gathered}
$$

while $\sigma$ has been fixed in both equations for simplicity. The spectrum of the linear combination is:

$$
\begin{aligned}
C_{k, k+1}(f) & =a_{1} P_{k}(f)+a_{2} P_{k+1}(f) \\
& =(2 \pi f)^{k} j^{k} e^{-2(\pi f \sigma)^{2}}\left[a_{1} A_{1}+j a_{2} A_{2}(2 \pi f)\right]
\end{aligned}
$$

where $a_{1}$ and $a_{2}$ are the nonzero weighting coefficients. Hence, the power spectral density becomes:

$$
\begin{gathered}
S_{k, k+1}(f)=\left|C_{k, k+1}(f)\right|^{2} \\
=(2 \pi f)^{2 k} e^{-4(\pi f \sigma)^{2}}\left[\left(a_{1} A_{1}\right)^{2}+\left(a_{2} A_{2}\right)^{2}(2 \pi f)^{2}\right]
\end{gathered}
$$

The peak frequency can be obtained by developing the frequency derivative $S_{k, k+1}^{\prime}(f)$ formula, then solving $S_{k, k+1}^{\prime}(f)=0$. After several mathematical steps, and taking into account finite nonzero frequencies, we reach the following:

$$
\left[k-(2 \pi f)^{2} \sigma^{2}\right]\left[\left(a_{1} A_{1}\right)^{2}+(2 \pi f)^{2}\left(a_{2} A_{2}\right)^{2}\right]+\left(a_{2} A_{2}\right)^{2}(2 \pi f)^{2}=0
$$

$A_{1}$ and $A_{2}$ determine the energy of $P_{k}(f)$ and $P_{k+1}(f)$ respectively, which has to be conserved for both pulses before being combined. The energy expressions 
of $P_{k}(f)$ and $P_{k+1}(f)$ are given as:

$$
\begin{array}{r}
E\left\{P_{k}\right\}=\int_{-\infty}^{+\infty}\left|P_{k}(f)\right|^{2} d f=\int_{-\infty}^{+\infty} A_{1}^{2}(2 \pi f)^{2 k} e^{-4(\pi f \sigma)^{2}} \mathrm{df} \\
E\left\{P_{k+1}\right\}=\int_{-\infty}^{+\infty}\left|P_{k+1}(f)\right|^{2} d f=\int_{-\infty}^{+\infty} A_{2}^{2}(2 \pi f)^{2 k+2} e^{-4(\pi f \sigma)^{2}} \mathrm{df}
\end{array}
$$

Apply integration by parts for equation (12):

$$
E\left\{P_{k+1}\right\}=\frac{(2 k+1) 2 \pi}{4 \pi \sigma^{2}} \int_{-\infty}^{+\infty} A_{2}^{2}(2 \pi f)^{2 k} e^{-4(\pi f \sigma)^{2}} \mathrm{df}
$$

To keep on same energy level, state $E\left\{P_{k}\right\}=E\left\{P_{k+1}\right\}$, then by referring to equations (11) and (13):

$$
A_{1}=\sqrt{\frac{(2 k+1)}{2 \sigma^{2}}} A_{2}
$$

Substitute $A_{1}$ in equation (10), given that $A_{2} \neq 0$, therefore

$$
\left[k-(2 \pi f)^{2} \sigma^{2}\right]\left[a_{1}^{2} \frac{(2 k+1)}{2 \sigma^{2}}+(2 \pi f)^{2} a_{2}^{2}\right]+\left[a_{2}^{2}(2 \pi f)^{2}\right]=0
$$

Let $u=(2 \pi f)^{2}$, then simplify to obtain:

$$
-2 \sigma^{2} a_{2}^{2} u^{2}+\left(2 k a_{2}^{2}-a_{1}^{2}(2 k+1)+2 a_{2}^{2}\right) u+k a_{1}^{2} \frac{(2 k+1)}{\sigma^{2}}=0
$$

Let $a_{2}=\beta a_{1}$, while $\beta \neq 0$, hence

$$
-2 \sigma^{2} \beta^{2} u^{2}+\left(2 k \beta^{2}-2 k-1+2 \beta^{2}\right) u+k \frac{(2 k+1)}{\sigma^{2}}=0
$$

It is a second degree equation with:

$$
\Delta=4 \beta^{4}(k+1)^{2}+(2 k+1)^{2}+4 \beta^{2}(2 k+1)(k-1)
$$

Based on the fact that $k$ is a nonzero natural number, then for a positive root value:

$$
u=\frac{k+1}{2 \sigma^{2}}-\frac{2 k+1}{4 \sigma^{2} \beta^{2}}+\sqrt{\frac{(k+1)^{2}}{4 \sigma^{4}}+\frac{(2 k+1)^{2}}{16 \sigma^{4} \beta^{4}}+\frac{(2 k+1)(k-1)}{4 \beta^{2} \sigma^{4}}}
$$

Therefore, by taking into account the positive band,

$$
f=f_{\text {peak }}=\frac{1}{4 \pi \sigma} \times
$$




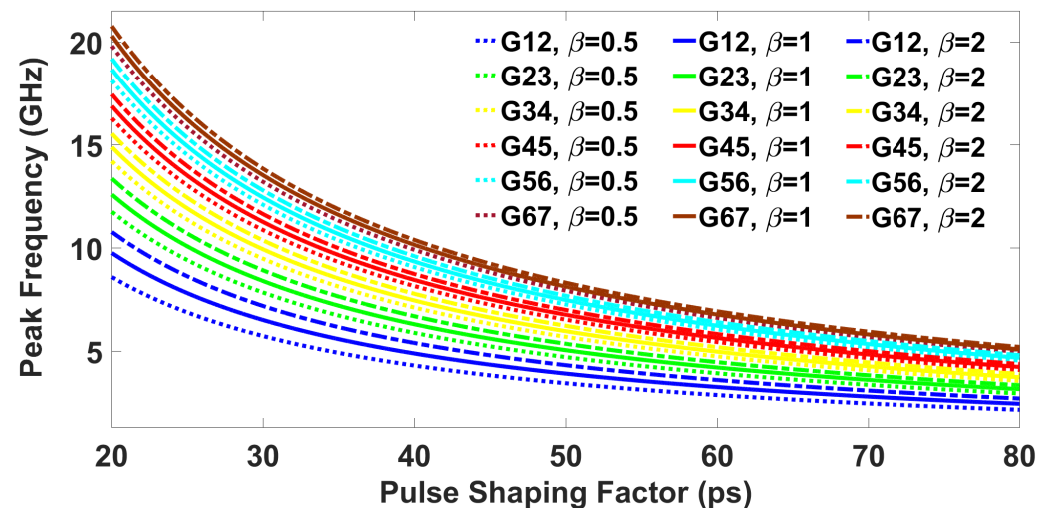

Fig. 3 Peak frequency versus pulse shaping factor $(\sigma)$ for the linear combinations of $k^{\text {th }}$ and $(k+1)^{t h}$ Gaussian derivatives (denoted as $G[k][k+1]$ ), while considering different values of $\beta$.

$$
\sqrt{2(k+1)-\frac{2 k+1}{\beta^{2}}+\sqrt{4(k+1)^{2}+\frac{(2 k+1)^{2}}{\beta^{4}}+\frac{4(2 k+1)(k-1)}{\beta^{2}}}}
$$

Considering different values of $\beta$, figure (3) plots the peak frequency versus the pulse shaping factor for numerous couple combinations of Gaussian derivatives $(1 \leq k \leq 6)$. The figure generally illustrates an inverse relation between $\sigma$ and peak frequency, while the latter also increases with the order of derivation $k$, since a time differentiator acts as a high pass filter. Moreover, $\beta$ determines the weight of the $(k+1)^{t h}$ pulse inside the combination, which further increases the peak frequency for $\beta \geq 1$. The power spectral density (PSD) plots for the above waveforms are displayed in figure (4), for $\sigma=50$ ps and $\beta=1$, where the spectra are scaled to the maximum radiation limit of FCC. The peak frequency well indicates the spectrum compliance with the regular mask, besides to the $10 \mathrm{~dB}$ bandwidth which determines the utilization of the spectral margin under emission standard. A legal transmission requires to adapt the spectra shown in figure (4) with FCC, that has a direct influence on the signal power for most waveforms, hence, a fine selection for the candidate combination would be important to collect the maximum possible energy without violating the limit.

As a time domain interpretation, figure (5) points out that the zero crossings and multi-peaks appearing in the waveforms relying on greater orders of derivation shift the corresponding spectra towards higher frequencies. All pulse combinations are defined over a time slot of $0.5 \mathrm{~ns}$, thus promising for a maximum achievable data rate of 2 Gbps. Figures (3),(4), and (5), show that the pulse shaping factor, weighting coefficient, and derivation order, are key variables which control the time and spectral response of the generated signal. In the next section, we will use the previously introduced model to design UWB combinations while involving the previously delineated parameters, in 


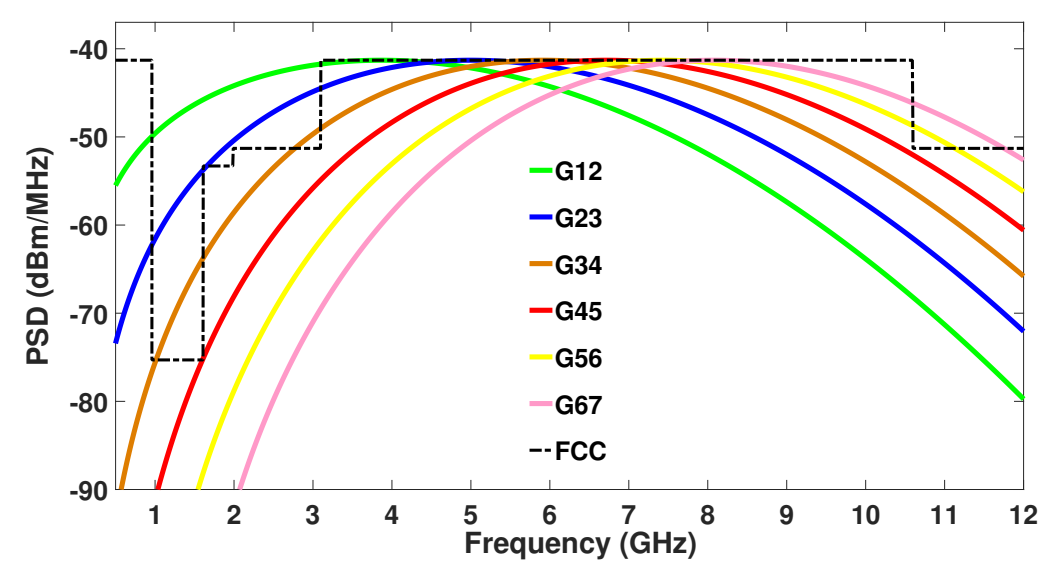

Fig. 4 The power spectral density for the linear combinations of $k^{t h}$ and $(k+1)^{t h}$ Gaussian derivatives, with $\beta=1$ and $\sigma=50$ ps.

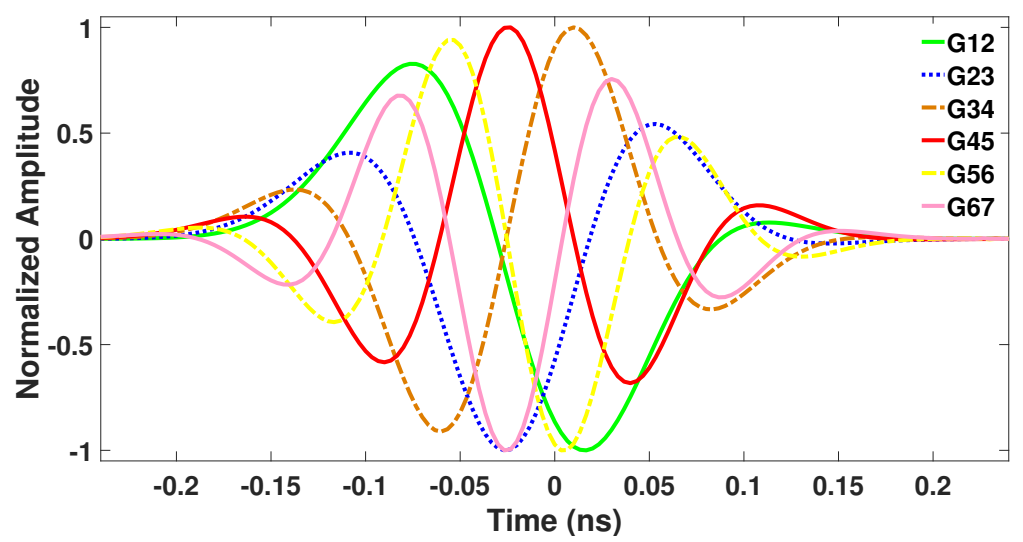

Fig. 5 The time-domain waveforms for the linear combinations of $k^{\text {th }}$ and $(k+1)^{t h}$ Gaussian derivatives, with $\beta=1$ and $\sigma=50$ ps.

addition to the time delay between elementary pulses which is shown to be another critical parameter in shaping the target waveform.

\section{Novel Optimized Pulse Designs}

In this section, we present four novel UWB pulses that have been obtained by global search using the optimization procedure described in figure (2). Initially, $\sigma$ has been fixed for the basic waveforms to avoid using separate pulse generators, thus, reduce the system complexity. Then, different values of $\sigma$ have been eventually considered (subsection 5.4) to reach out the highest possible efficiency, at the expense of a more complex design. 

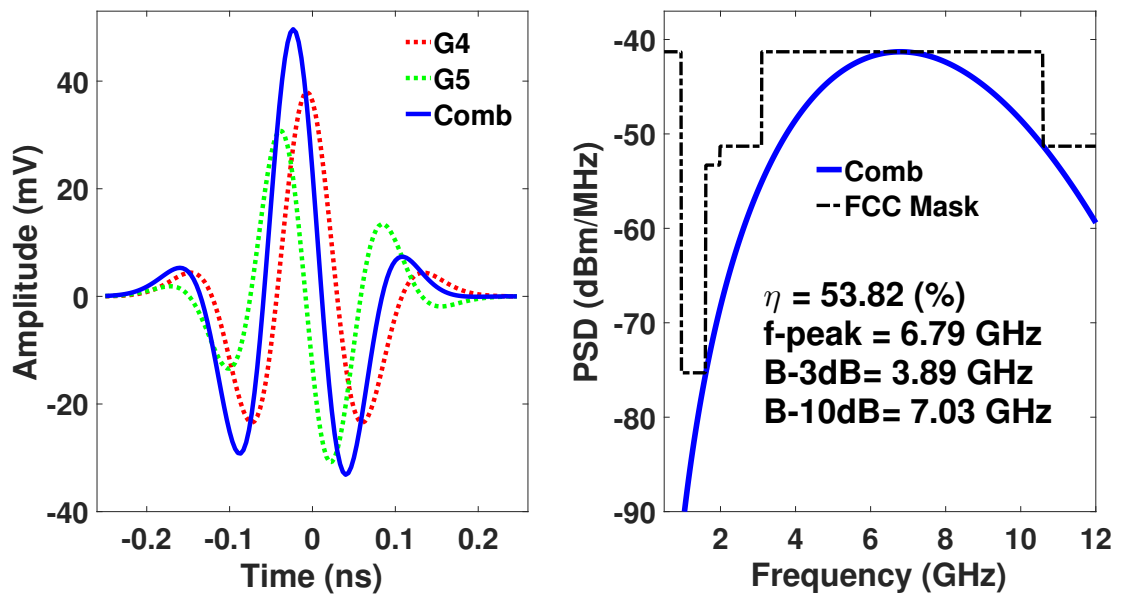

Fig. 6 Time waveforms of the $4^{\text {th }}$ and $5^{\text {th }}$ Gaussians besides to their linear combination (left side), in addition to the resultant spectrum under FCC mask (right side), reaching an efficiency of $53.8 \%$. G4 $=4^{\text {th }}$ Gaussian, G5 $=5^{\text {th }}$ Gaussian, Comb= combination.

\subsection{The $4^{\text {th }}-5^{\text {th }}$ Couple Combination based on same Pulse Shaping Factor}

In this subsection, we show that the $4^{t h}-5^{t h}$ couple is a good choice for an improved power efficiency at limited order of complexity. The $4^{t h}-5^{t h}$ is a special case of the $[k, k+1]^{t h}$ general combination form, hence, to find the peak frequency we fix $\mathrm{k}=4$ in equation (20) resulting in

$$
f_{\text {peak }}=\frac{1}{4 \pi \sigma} \sqrt{10-\frac{9}{\beta^{2}}+\sqrt{100+\frac{81}{\beta^{4}}+\frac{108}{\beta^{2}}}}
$$

The optimal settings for the proposed combination obtained through simulations are $\sigma=49.09 \mathrm{ps}$ and $\beta=0.89$. The time waveforms and resultant spectrum are plotted in figure (6), while an efficiency of $53.8 \%$ has been recorded. To a certain extent, the derivation order determines the frequency location of spectrum energy, hence, using consecutive orders of derivation would slightly broaden the aimed spectral area under the radiation limit. Our developed combination is much simpler than the one investigated in [53], as the former is based on the same pulse shaping factor for both pulses, thus requiring only a single pulse generator.

\subsection{Dual $4^{\text {th }}$ Combination based on same Pulse Shaping Factor}

Besides to the $4^{t h}-5^{t h}$ Gaussian couple, it has been also realized that two identical $4^{\text {th }}$ Gaussians can construct an alternative waveform by introducing a time delay $\delta$ in between. The spectrum of this combination can be expressed 

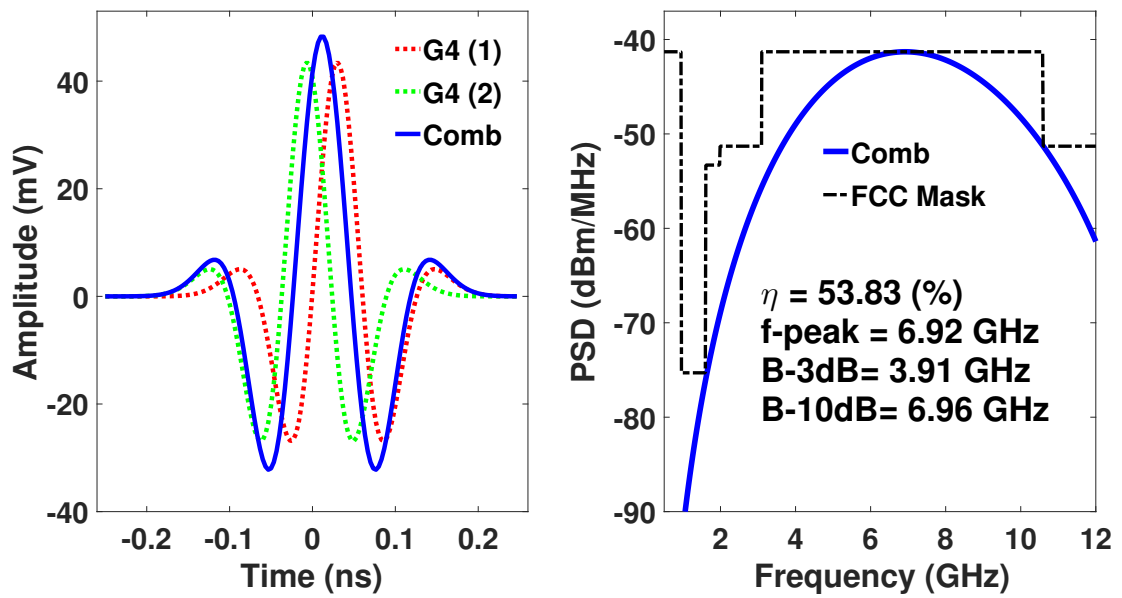

Fig. 7 Time waveforms of the two $4^{\text {th }}$ Gaussians and their linear combination (left side), besides to the resultant spectrum under FCC mask (right side), reaching an efficiency of 53.8 \%. G4 (1) = First $4^{\text {th }}$ Gaussian, G4 (2) $=$ Second Shifted $4^{\text {th }}$ Gaussian, Comb= combination.

as:

$$
C_{4,4}(f)=a_{1} P_{4}(f)+a_{2} P_{4}(f) e^{-j 2 \pi f \delta}
$$

Both pulses are identical, then $a_{1}=a_{2}=a$, hence,

$$
\begin{gathered}
C_{4,4}(f)=a P_{4}(f)\left(1+e^{-j 2 \pi f \delta}\right)=a A_{4}(2 \pi f j)^{4} e^{-2(\pi f \sigma)^{2}}\left(1+e^{-j 2 \pi f \delta}\right) \\
=a A_{4}(2 \pi f)^{4} e^{-2(\pi f \sigma)^{2}} e^{-j \pi f \delta} 2 \cos (\pi f \delta)
\end{gathered}
$$

Then, the power spectral density can be expressed as

$$
S_{4,4}(f)=\left|C_{4,4}(f)\right|^{2}=a^{2} A_{4}^{2}(2 \pi f)^{8} e^{-4(\pi f \sigma)^{2}} 4 \cos ^{2}(\pi f \delta)
$$

As in section 4, we follow a similar mathematical procedure to find the peak frequency, as clarified in Appendix A:

$$
f_{\text {peak }}=\frac{1}{\pi} \sqrt{\frac{3\left(3 \delta^{2}+4 \sigma^{2}\right)-\sqrt{3\left(19 \delta^{4}-24 \delta^{2} \sigma^{2}+48 \sigma^{4}\right)}}{\delta^{2}\left(12 \sigma^{2}+\delta^{2}\right)}}
$$

Figure (7) presents the time and frequency plots of this dual combination for $\sigma=41 \mathrm{ps}$ and $\delta=37 \mathrm{ps}$ extracted by optimization. The oscillating term involved in equation (24) has a positive shaping effect on the spectrum of $4^{\text {th }}$ Gaussian, so the latter well suits FCC mask and the recorded efficiency is almost the same as the previous combination $(53.8 \%)$. The maximum achievable efficiency of single $4^{\text {th }}$ Gaussian pulse obtained in simulations is $49.3 \%$, so it could be concluded that the performance of $4^{\text {th }}$ Gaussian can be 


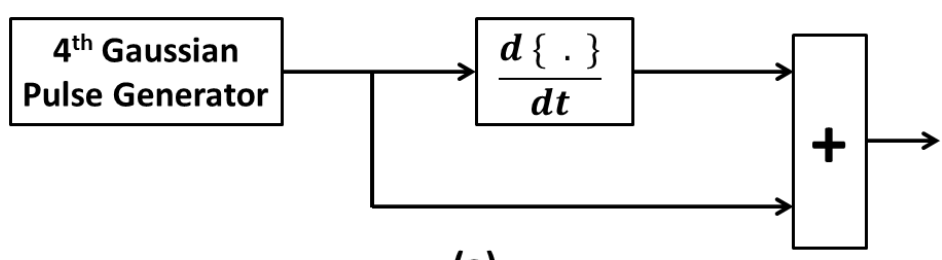

(a)

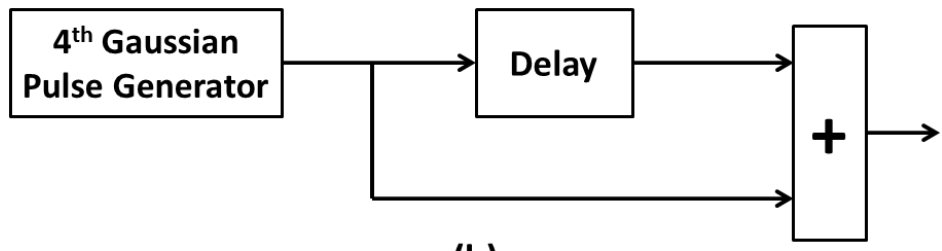

(b)

Fig. 8 Block diagram for the generation of the $4^{t h}-5^{t h}$ combination (a) and dual $4^{t h}$ combination (b).

notably enhanced by either adding a simple differentiator or a delay line, as described in figure (8).

\subsection{The $5^{t h}-6^{t h}$ Couple Combination based on the same Pulse Shaping Factor}

Here, we develop a new combination relying on higher orders of Gaussian derivative, the $5^{\text {th }}$ and $6^{\text {th }}$ pulses that can constitute a good basis for composing a more efficient waveform, using a time delay. The spectra of the utilized pulses are $P_{5}(f)$ and $P_{6}(f)$ which can be expressed as:

$$
\begin{gathered}
P_{5}(f)=j A_{5}(2 \pi f)^{5} e^{-2(\pi f \sigma)^{2}} \\
P_{6}(f)=-A_{6}(2 \pi f)^{6} e^{-2(\pi f \sigma)^{2}}
\end{gathered}
$$

where $A_{5}$ and $A_{6}$ are the amplitudes of the $5^{\text {th }}$ and $6^{\text {th }}$ Gaussian derivatives respectively ( $A_{6}=\sqrt{\frac{2}{11}} \sigma A_{5}$ to equalize energies), and $\sigma$ has been also fixed for both waveforms to keep on low implementation complexity. The $5^{\text {th }}$ Gaussian pulse is delayed by $\delta$, then the resultant spectrum for the linear combination is:

$$
C_{5,6}(f)=a_{5} P_{5}(f) e^{-j 2 \pi f \delta}+a_{6} P_{6}(f)
$$

while $a_{5}$ and $a_{6}$ are the weighting coefficients for the $5^{\text {th }}$ and $6^{\text {th }}$ pulses respectively, hence

$$
C_{5,6}(f)=(2 \pi f)^{5} e^{-2(\pi f \sigma)^{2}} \times
$$




$$
\left[a_{5} j A_{5} \cos (2 \pi f \delta)+a_{5} A_{5} \sin (2 \pi f \delta)-a_{6} A_{6}(2 \pi f)\right]
$$

So the power spectral density is

$$
\begin{gathered}
S_{5,6}(f)=\left|C_{5,6}(f)\right|^{2}=(2 \pi f)^{10} e^{-4(\pi f \sigma)^{2}} \times \\
{\left[\left(a_{5} A_{5} \cos (2 \pi f \delta)\right)^{2}+\left(a_{5} A_{5} \sin (2 \pi f \delta)-a_{6} A_{6}(2 \pi f)\right)^{2}\right]}
\end{gathered}
$$

Substitute $A_{6}$ by $\sqrt{\frac{2}{11}} \sigma A_{5}$, then simplify:

$$
\begin{gathered}
S_{5,6}(f)=(2 \pi f)^{10} e^{-4(\pi f \sigma)^{2}} A_{5}{ }^{2} \times \\
{\left[a_{5}{ }^{2}-2 a_{5} \sin (2 \pi f \delta) a_{6} \sqrt{\frac{2}{11}} \sigma(2 \pi f)+a_{6}{ }^{2} \frac{2}{11} \sigma^{2}(2 \pi f)^{2}\right]}
\end{gathered}
$$

The filtering effect exhibited in the right term of equation (31) seems to be promising in designing the spectrum of our novel combination. Similarly as before, to get the peak frequency we have to solve $S_{5,6}^{\prime}(f)=0$. After several mathematical derivations provided in Appendix B, while defining $U=(2 \pi f)^{2}$, we reach the following equation:

$$
K_{1} U^{3}+K_{2} U^{2}+K_{3} U+K_{4}=0
$$

where,

$$
\begin{gathered}
K_{1}=a_{6} \sqrt{\frac{2}{11}} \delta^{3} \sigma\left[-\delta^{2} / 24-\sigma^{2} / 3\right] \\
K_{2}=\sqrt{\frac{2}{11}} a_{6} \sigma\left[14 \delta^{3} / 6+2 \sigma^{2} \delta-a_{6} \sqrt{\frac{2}{11}} \sigma^{3}\right] \\
K_{3}=-12 a_{6} \sqrt{\frac{2}{11}} \sigma \delta+6 a_{6}{ }^{2} \frac{2}{11} \sigma^{2}-\sigma^{2} \\
K_{4}=5
\end{gathered}
$$

We notice in (32) a third degree polynomial equation that can be solved based on Cardano's method:

$$
\begin{gathered}
\Delta_{0}=K_{2}{ }^{2}-3 K_{1} K_{3} \\
\Delta_{1}=2 K_{2}{ }^{3}-9 K_{1} K_{2} K_{3}+27 K_{1}{ }^{2} K_{4}
\end{gathered}
$$

Hence

$$
U=-\frac{1}{3 K_{1}}\left(K_{2}+\chi+\frac{\Delta_{0}}{\chi}\right)
$$

while

$$
\chi=\sqrt[3]{\frac{\Delta_{1} \pm \sqrt{\Delta_{1}^{2}-4 \Delta_{0}{ }^{3}}}{2}}
$$

Even if $\sigma$ is not the only parameter controlling the peak frequency, especially when time delay is involved in the combination, we can still deduce from figure (3) the range of $\sigma$ where $f_{\text {peak }}$ lies at the mid interval of UWB allocated 
band. It can be noticed that for the $5^{\text {th }}-6^{\text {th }}$ couple, $\sigma$ is mainly between 50 ps and 60 ps. Besides, in a dual linear combination, it is preferred to avoid a significant difference between the weighting factor magnitudes of the basic pulses utilized, in order to keep on comparable energy levels and allow both pulses to play a major role in designing the target waveform. As $a_{5}$ has been fixed at 1 (Appendix B), then $a_{6}$ is assumed not to exceed 3 so that it is not significantly larger than $a_{5}$. For the time delay, similarly as before, $\delta$ is assumed to be not greater than 50 ps (Appendix A). Hence, based on the probable values of $\sigma, a_{6}$, and $\delta$; we estimate $K_{1}, K_{2}$, and $K_{3}$, to approximate the above complicated terms, and finally we obtained that:

$\Delta_{0}<0$ and $\Delta_{1}>0$, while both $\Delta_{0}$ and $\Delta_{1}$ are absolutely far below 1 . It has been also concluded that: $\Delta_{1} \ll 2\left|\Delta_{0}\right|^{\frac{3}{2}}$, then $\Delta_{1}{ }^{2} \lll 4\left|\Delta_{0}\right|^{3}$. Therefore,

$$
\begin{aligned}
\chi & =\sqrt[3]{\frac{\Delta_{1} \pm \sqrt{\Delta_{1}^{2}+4\left|\Delta_{0}\right|^{3}}}{2}} \approx \sqrt[3]{\frac{\Delta_{1} \pm \sqrt{4\left|\Delta_{0}\right|^{3}}}{2}} \\
& =\sqrt[3]{\frac{\Delta_{1} \pm 2\left|\Delta_{0}\right|^{\frac{3}{2}}}{2}} \approx \sqrt[3]{ \pm\left|\Delta_{0}\right|^{\frac{3}{2}}}=\sqrt{\left|\Delta_{0}\right|}
\end{aligned}
$$

Hence

$$
U=-\frac{1}{3 K_{1}}\left(K_{2}+\sqrt{\left|\Delta_{0}\right|}+\frac{\Delta_{0}}{\sqrt{\left|\Delta_{0}\right|}}\right) \approx-\frac{K_{2}}{3 K_{1}}
$$

Therefore

$$
U=\frac{14 \delta^{3} / 6+2 \sigma^{2} \delta-a_{6} \sqrt{\frac{2}{11}} \sigma^{3}}{3 \delta^{3}\left(\delta^{2} / 24+\sigma^{2} / 3\right)}
$$

Implying that

$$
f=f_{\text {peak }}=\sqrt{\frac{7 \delta^{3} / 3+2 \sigma^{2} \delta-a_{6} \sqrt{\frac{2}{11}} \sigma^{3}}{\pi^{2} \delta^{3}\left(\delta^{2} / 2+4 \sigma^{2}\right)}}
$$

The spectrum shape has to be altered in order to best fit the emission limit, hence, the pulses parameters have to be optimized. By employing the interior point method, we have reached the optimal settings in the sense of maximizing the efficiency under FCC: $\sigma=57.72 \mathrm{ps}, a_{6}=1.46, \delta=30 \mathrm{ps}$. The time waveforms and corresponding spectra of the $5^{\text {th }}$ Gaussian, $6^{\text {th }}$ Gaussian, besides to the proposed combination, are plotted in figure (9). A power efficiency of around $65 \%$ has been recorded, which is relatively large with respect to that of previous signals. Thanks to the high degree of freedom offered by the multivariable combination, besides to the greater order of derivation for utilized Gaussians, it was possible to adequately design the spectrum. The shape of the 



Fig. 9 The time waveforms of the $5^{\text {th }}$ and $6^{\text {th }}$ Gaussians besides to their linear combination (left side), in addition to the resultant spectrum under FCC mask (right side), reaching an efficiency of around $65 \%$. G5 $=5^{\text {th }}$ Gaussian, G6 $=6^{\text {th }}$ Gaussian, Comb $=$ combination.

latter has been adapted by enlarging the 3 - $\mathrm{dB}$ bandwidth while fixing the 10$\mathrm{dB}$ bandwidth at around 7.5 GHz, exploiting thus a wide spectral area under FCC without violating the regular limit. Figure (10) describes the generation stages for the current combination, which appears to be more complex than the $4^{t h}-5^{t h}$ and dual $4^{\text {th }}$ combinations, following from integrating a differentiator and a delay line simultaneously, in addition to the increased derivation order of original pulses used.

Figure (9) illustrates that the value of $f_{\text {peak }}$ computed analytically $(6.25 \mathrm{GHz})$ based on equation (40) lies in the peak region, as a spread in the spectrum appears at the peak, however, the exact peak frequency measured in simulation is around $6.86 \mathrm{GHz}$. The difference is due to the approximations done, taking into consideration that it was challenging to continue with the precise analytical forms through the mathematical development. Such deviation is judged to be acceptable, since at $6.25 \mathrm{GHz}$ the measured value for the spectrum is very close to the peak.

\subsection{Triplet Combination based on One $6^{\text {th }}$ and two $7^{\text {th }}$ Gaussians with different Pulse Shaping Factors}

The previously designed waveforms show a good potential which is limited by the system requirements, while taking into account the operation at relatively low orders of complexity. Let us here prioritize the power efficiency and allow an acceptable complication for the pulse generation technique. Using three distinct Gaussian pulses with independent parameters, we can shape the target waveform by exploiting the highest degree of freedom, due to the increased number of variables involved in the combination. The general form of the 


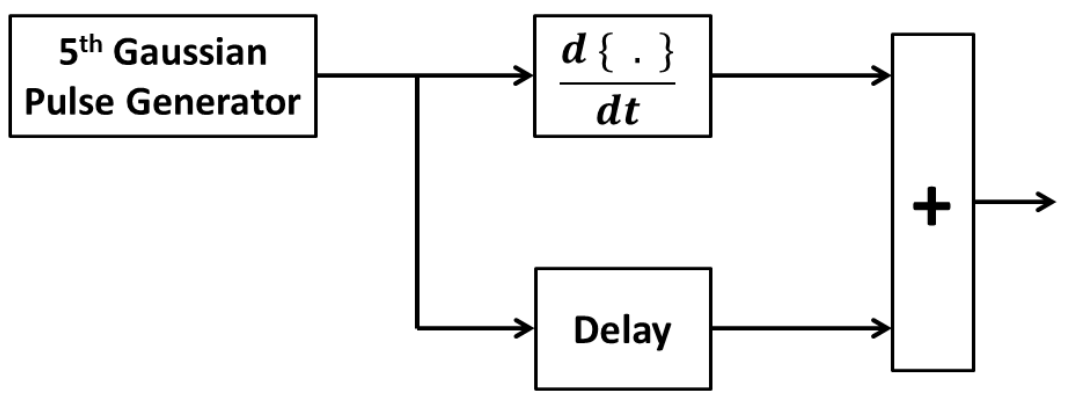

Fig. 10 Block diagram for the generation of the $5^{t h}-6^{t h}$ combination.


Fig. 11 The time waveforms of the $6^{\text {th }}$ Gaussian and two $7^{\text {th }}$ Gaussians besides to their linear combination (left side), in addition to the resultant spectrum under FCC mask (right side), reaching an efficiency of around $72 \%$. G6 $=6^{\text {th }}$ Gaussian, G7 (1) $=$ First $7^{\text {th }}$ Gaussian, G7 (2) $=$ Second $7^{\text {th }}$ Gaussian.

proposed pulse spectrum can be expressed as:

$$
C_{k_{1}, k_{2}, k_{3}}(f)=a_{1} P_{k_{1}, \sigma_{1}}(f)+a_{2} P_{k_{2}, \sigma_{2}}(f)+a_{3} P_{k_{3}, \sigma_{3}}(f)
$$

By running the global optimization scheme we extract the best parameters' values which can be set to reach the most fitting spectrum with FCC mask. The simulation outcomes have been obtained as: $k_{1}=6, k_{2}=7, k_{3}=7, a_{1}=1$, $a_{2}=-1.94, a_{3}=1.13, \sigma_{1}=75.58 \mathrm{ps}, \sigma_{2}=48.37 \mathrm{ps}, \sigma_{3}=38 \mathrm{ps}$.

The resultant spectrum can be formulated as:

$$
C_{6,7,7}(f)=a_{1} A_{6}(2 \pi f j)^{6} e^{-2\left(\pi f \sigma_{1}\right)^{2}}+a_{2} A_{7}(2 \pi f j)^{7} e^{-2\left(\pi f \sigma_{2}\right)^{2}}
$$




$$
+a_{3} A_{7}(2 \pi f j)^{7} e^{-2\left(\pi f \sigma_{3}\right)^{2}}
$$

Hence, the power spectral density becomes:

$$
\begin{gathered}
S_{6,7,7}(f)=\left|C_{6,7,7}(f)\right|^{2}=a_{1}{ }^{2} A_{6}{ }^{2}(2 \pi f)^{12} e^{-4\left(\pi f \sigma_{1}\right)^{2}} \\
+A_{7}^{2}(2 \pi f)^{14}\left(a_{2} e^{-2\left(\pi f \sigma_{2}\right)^{2}}+a_{3} e^{-2\left(\pi f \sigma_{3}\right)^{2}}\right)^{2}
\end{gathered}
$$

The opposite signs for $a_{2}$ and $a_{3}$ have a shaping effect on the right exponential term, while the powers of frequency at different degrees play a role in filtering the spectrum to well match the regulation limit. The peak frequency has not been derived as it is difficult to obtain its explicit formula when dealing with multiple dissimilar exponential functions.

Figure (11) displays the time waveforms of the basic pulses (one $6^{\text {th }}$ and two $7^{\text {th }}$ Gaussians) utilized besides to their linear combination and corresponding spectrum. A achieved power efficiency of $72 \%$ is extremely high for Gaussianbased waveforms, owing to the large number of independent pulse variables which enabled us to efficiently design the targeted combination. However, the latter achievement has been realized at the cost of complexity, as three separate parallel circuits are needed to synthesize a triplet coupling $\left(\sigma_{1} \neq \sigma_{2} \neq \sigma_{3}\right)$ which requires additional components in the generation system. To the best of our knowledge, $72 \%$ is an unprecedented spectral efficiency realized with analog carrier-free impulse radio, utilizing a few electrically generated pulses of the Gaussian family. It is also worth highlighting the fact that no signalprocessing units have been functionalized in our pulse shaping mechanisms, like FIR filters or wavelength-to-time converters ([24, 25, 47]), as well, no local oscillators have been used, nor extremely high-orders of Gaussian derivatives ([46]). So in this contribution, we took into account the IR system implementation issues while aiming to reach a simple and reduced-size for the targeted generator architecture in the real application.

\section{Performance Analysis and BER Evaluation in AWGN and Multi-path Channels}

Figure (12) represents the spectra of all proposed combinations, in addition to the $5^{\text {th }}$ Gaussian pulse $[37,39]$. Table 3 reports the specifications of the generated waveforms, while the frequency properties and power efficiencies are summarized in table 4 , besides to the peak-to-average power ratio (PAPR) measures. As mentioned earlier, it could be concluded that spreading the 3$\mathrm{dB}$ bandwidth $B_{3}$ is the reason behind the efficiency enhancement as long as the $10-\mathrm{dB}$ band is $B_{10} \leq 7.5 \mathrm{GHz}$, which is the frequency range allocated for UWB communications. A spectrum with $B_{10}=7.5 \mathrm{GHz}$ can exactly match the regular mask from upper boundary $(-41.3 \mathrm{dBm} / \mathrm{MHz})$ and lateral limits, while $B_{3}$ determines the spectral coverage area and definitely the collected energy under FCC. The increased number of variables inside a combination 


\begin{tabular}{|l|l|l|}
\multicolumn{1}{|c|}{ Generated Waveforms } & $\begin{array}{l}\text { Number of } \\
\text { Pulses Used }\end{array}$ & Pulse/s Parameters \\
\hline $5^{t h}$ single & 1 & $\sigma=51 \mathrm{ps}$ \\
\hline $4^{t h}-5^{t h}$ Couple & 2 & $\sigma=49.09 \mathrm{ps}, \beta=0.89$ \\
\hline Dual $4^{\text {th }}$ Combination & 2 & $\sigma=41 \mathrm{ps}, \delta=37 \mathrm{ps}$ \\
\hline $5^{t h}-6^{\text {th }}$ Couple & 2 & $\begin{array}{l}\sigma=57.72 \mathrm{ps}, a_{5}=1, a_{6}= \\
1.46, \delta=30 \mathrm{ps}\end{array}$ \\
\hline $6^{\text {th }}$ and Dual $7^{\text {th }}$ Combination & 3 & $\begin{array}{l}a_{1}=1, a_{2}=-1.94, a_{3}=1.13, \\
\sigma_{1}=75.58 \mathrm{ps}, \sigma_{2}=48.37 \mathrm{ps}, \\
\sigma_{3}=38 \mathrm{ps}\end{array}$ \\
& &
\end{tabular}

Table 3 Parameters of the generated waveforms.

\begin{tabular}{|l|l|l|l|l|l|} 
Generated Waveforms & $\begin{array}{l}f_{\text {peak }} \\
(\mathrm{GHz})\end{array}$ & $\begin{array}{l}B_{3} \\
(\mathrm{GHz})\end{array}$ & $\begin{array}{l}B_{10} \\
(\mathrm{GHz})\end{array}$ & $\eta(\mathbf{\%})$ & $\begin{array}{l}\text { PAPR } \\
\mathbf{( d B})\end{array}$ \\
\hline $5^{\text {th }}$ single & 6.98 & 3.65 & 6.61 & 50.71 & 7.95 \\
\hline $4^{\text {th }}-5^{\text {th }}$ Couple & 6.79 & 3.89 & 7.03 & 53.82 & 9.06 \\
\hline Dual $4^{\text {th }}$ Combination & 6.92 & 3.91 & 6.96 & 53.83 & 8.83 \\
\hline $5^{\text {th }}-6^{\text {th }}$ Couple & 6.86 & 4.88 & 7.5 & 64.95 & 8.65 \\
\hline $6^{\text {th }}$ and Dual $7^{\text {th }}$ Combination & 7.72 & 5.45 & 7.5 & 72.02 & 7.74
\end{tabular}

Table 4 Frequency properties and power efficiencies of the generated waveforms $\left(f_{\text {peak }}\right.$ : peak frequency, $B_{3}: 3 \mathrm{~dB}$ band, $B_{10}: 10 \mathrm{~dB}$ band, $\eta$ : power efficiency), besides to the peak-to-average power ratio (PAPR).

leads to a superior control of the signal dynamics and consequently the spectral characteristics of the resultant waveform. Tables 3 and 4 inform that the performance gain occurs at the expense of complexity, as a satisfactory power efficiency can be achieved by applying the $4^{\text {th }}-5^{\text {th }}$ couple or dual $4^{\text {th }}$ Gaussian combination, with the advantage of requiring a single pulse generator besides to a simple differentiator or delay line, respectively. In contrast, a significant increase in the efficiency can be earned using higher orders of Gaussian derivatives, particularly one $6^{\text {th }}$ and two $7^{\text {th }}$ Gaussians, at the expense of employing three independent pulse generation circuits. The $5^{\text {th }}-6^{\text {th }}$ couple shows a good compromise between efficiency and complexity, which seems to be a promising solution for low-cost short-range impulse radio transmission.

The PAPR is an important criterion used to evaluate the pulses performance in presence of nonlinear effects inherent to electronic devices, like power amplifiers or signal converters [54-56]. The $4^{\text {th }}-5^{\text {th }}$ couple and dual $4^{\text {th }}$ combination show a poor performance in terms of PAPR, while the $6^{t h}-7^{t h}-7^{t h}$ triplet seems 


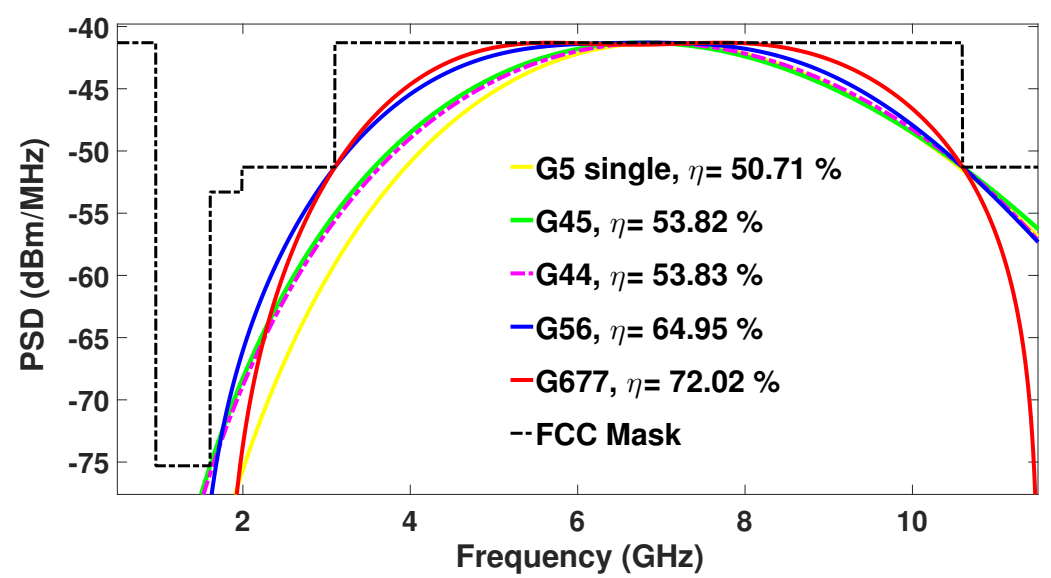

Fig. 12 The power spectral densities of the proposed pulse combinations, besides to the $5^{\text {th }}$ Gaussian pulse.
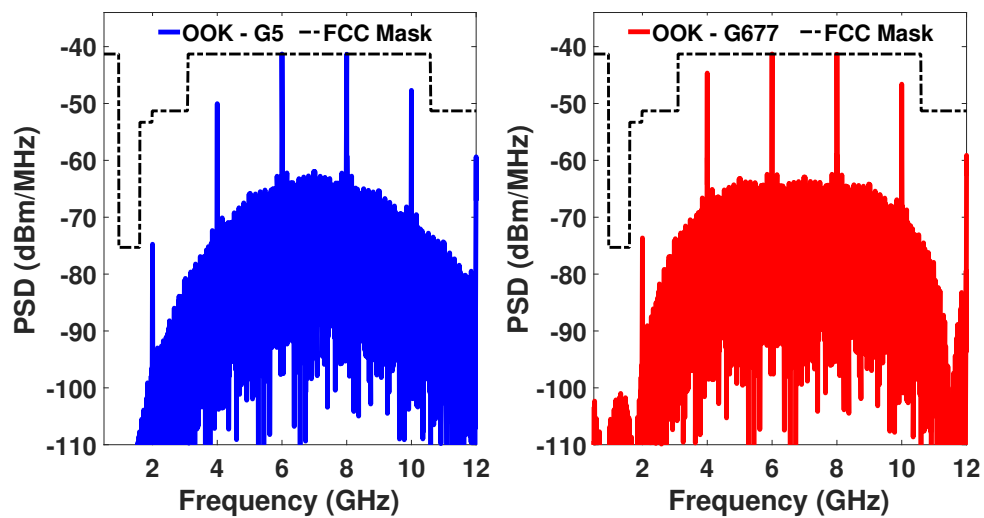

Fig. 13 Power spectral density for 2 Gbps On Off Keying based on the $5^{\text {th }}$ single pulse (left) and $6^{t h}-7^{t h}-7^{t h}$ triplet combination (right).

to be the best waveform in the sense of a minimized PAPR ratio, which is even better than $5^{\text {th }}$ single Gaussian. Similarly as the previously reported studies in section two, our proposed waveforms can be practically designed following the same generation scenario, and that brings a great promise for CMOS-based IR-UWB systems.

To evaluate the performance in terms of the data modulated impulse radio, two non-coherent transmission schemes have been applied, On Off Keying (OOK) and Pulse Position Modulation (PPM) [57]. Figure (13) describes the power spectral density of 2 Gbps OOK, while the utilized pulses are the $5^{\text {th }}$ single Gaussian (left) and $6^{t h}-7^{t h}-7^{t h}$ combination (right). The zero-power spectral spikes appear at multiples of the data rate, and the enhancement at the level 


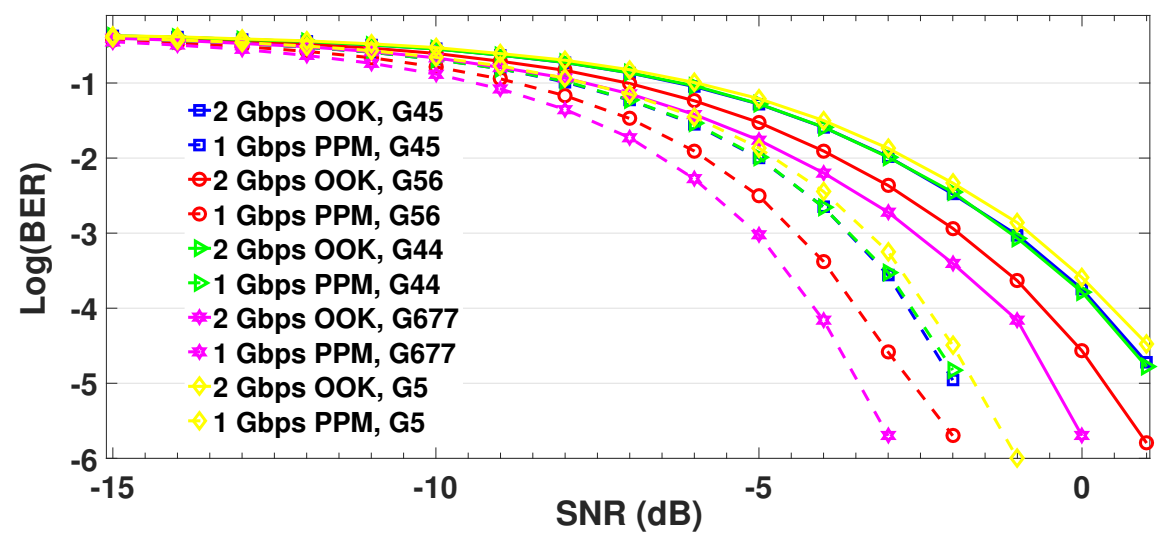

Fig. 14 The bit error rate for 2 Gbps OOK and 1 Gbps PPM based on different pulse shapes in AWGN channel.

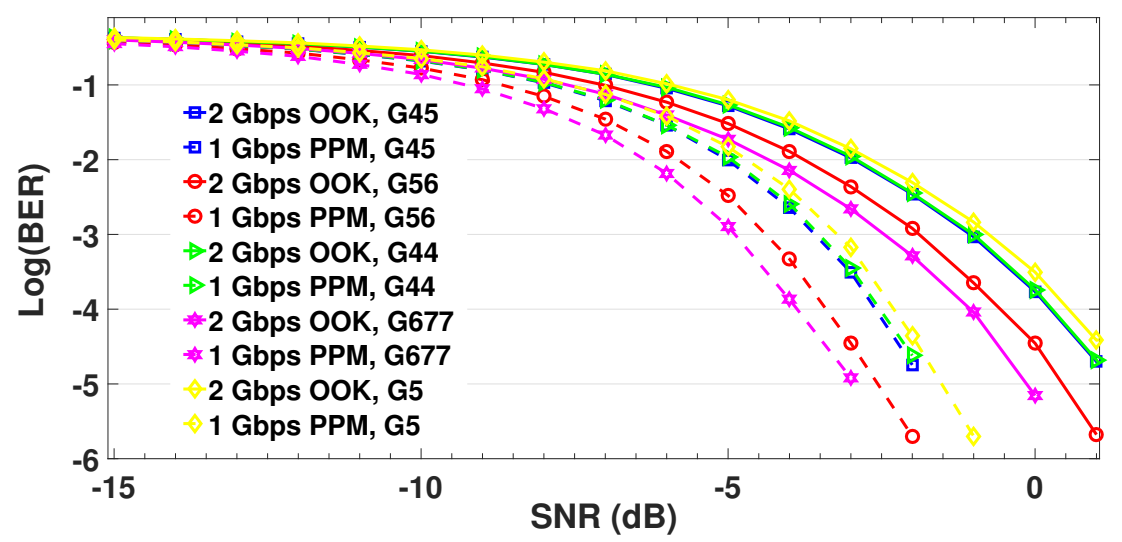

Fig. 15 The bit error rate for 2 Gbps OOK and 1 Gbps PPM based on different pulse shapes in multi-path fading channel.

of the pulse reflects on the whole modulation. As the envelope of these discrete lines takes the shape of basic pulse spectrum, hence, a wider area is covered by the $6^{\text {th }}-7^{\text {th }}-7^{t h}$ triplet in comparison to the $5^{\text {th }}$ single [58]. To further improve the spectral response, a Direct Sequence randomization method can be adopted to almost eliminate the frequency comb lines and shift up the spectrum towards FCC bound [59].

Figure (14) studies the bit error rate of 2 Gbps OOK and 1 Gbps PPM used with our candidate pulses in Additive White Gaussian Noise (AWGN) channel. It is obvious that increasing the power efficiency leads to a consequent reduction in the error probability, as a higher energy is transmitted to send a data symbol. A lower number of errors has been obtained with more efficient waveforms, and all developed combinations outperform the single $5^{\text {th }}$ Gaussian pulse. A similar conclusion can be drawn with the multi-path scenario 
in figure (15), while assuming 3 independent paths with a Rayleigh random channel delay averaging $80 \mathrm{ps}$ (16\% of the pulse duration). OOK and PPM modulation techniques are mainly robust to multi-path effects, as the decision at the receiver side is based on energy detection, so the internal signal dynamics do not play a major role in estimating the transmitted binary data. Especially with Gaussian family, where the energy is more concentrated at the mid interval of the pulse period and less distributed near time boundaries, hence, a good potential appears at low levels of inter-symbol interference. A performance degradation is expected in dense multi-path environments, which may raise the demand for transmitted reference schemes to overcome channel effects [60]. The $6^{\text {th }}-7^{\text {th }}-7^{\text {th }}$ waveform emerges as the best candidate among all other options in both AWGN and multi-path channels, which constitutes a strong alternative for reliable IR-UWB communications.

\section{Conclusion}

To enhance the quality of service for impulse radio communications while satisfying the regulatory spectral constraints, a highly efficient transmission is needed. In this paper, we introduced four power-efficient waveforms which are synthesized by linear combinations of Gaussian pulses. A systematic optimization prototype for extracting the best pulses' parameters has been used, and high spectral efficiencies have been achieved with different levels of tradeoff between performance and complexity. A combination of only 3 Gaussian derivatives, which can be generated via basic electronic circuits, showed an accurate approximation to the FCC mask over a large frequency range, while occupying a wide spectral area under the emission standard with an efficiency exceeding $70 \%$. Bit error rate simulation results demonstrate the great potential of our novel IR-UWB designs in AWGN and multi-path propagation channels, for 2 Gbps OOK and 1 Gbps PPM schemes. Considering alternative pulse types, besides to other regulation masks, is a good opportunity to extend our research. Another future research direction is to assess the effectiveness of the optimally shaped pulses in realistic transmission environments, so as to further validate their feasibility in practically implemented UWB systems.

\section{Appendix A}

Apply frequency derivative to the power spectral density in equation (24), then $S_{4,4}^{\prime}(f)=$

$$
\begin{gathered}
4 a^{2} A_{4}^{2}\left[8(2 \pi f)^{7} 2 \pi+(2 \pi f)^{8}\left(-8 \pi^{2} f \sigma^{2}\right)\right] e^{-4(\pi f \sigma)^{2}} \cos ^{2}(\pi f \delta) \\
-4 a^{2} A_{4}^{2}(2 \pi f)^{8} e^{-4(\pi f \sigma)^{2}} 2 \cos (\pi f \delta) \sin (\pi f \delta) \pi \delta
\end{gathered}
$$


To find $f_{\text {peak }}$ state $S_{4,4}^{\prime}(f)=0$, given that for finite nonzero frequencies $4 a^{2} A_{4}^{2}(2 \pi f)^{7} e^{-4(\pi f \sigma)^{2}} \neq 0$, then

$$
\left[16 \pi-2 \pi f\left(8 \pi^{2} f \sigma^{2}\right)\right] \cos ^{2}(\pi f \delta)-2(2 \pi f) \cos (\pi f \delta) \sin (\pi f \delta) \pi \delta=0
$$

Hence, either $\cos (\pi f \delta)=0$, or

$$
\left[16 \pi-2 \pi f\left(8 \pi^{2} f \sigma^{2}\right)\right] \cos (\pi f \delta)-2(2 \pi f) \sin (\pi f \delta) \pi \delta=0
$$

It is assumed that the time delay $\delta$ should not exceed $10 \%$ of the pulse period (0.5 ns), in order to avoid a large truncation of the pulse energy, therefore, we assume that $\delta \leq 50 \mathrm{ps}$. The power has to be concentrated in the UWB allocated band $([3.1-10.6] \mathrm{GHz})$, and the peak frequency is expected to lie at the mid interval of the desired band, as the $10 \mathrm{~dB}$ bandwidth has to be relatively large to cover a wide spectral margin under the regular mask. Hence, for practical values of $\delta$ and $f, \cos (\pi f \delta)$ is different from 0 , while for equation (46) it is sufficient the third order of Taylor series to approximate the sine and cosine functions:

$$
\begin{aligned}
& \sin (\pi f \delta)=(\pi f \delta)-(\pi f \delta)^{3} / 6 \\
& \cos (\pi f \delta)=1-(\pi f \delta)^{2} / 2
\end{aligned}
$$

Following these approximations, equation (46) can be simplified to eventually reach a polynomial form:

$$
\begin{aligned}
& {\left[8 \pi-2 \pi f\left(4 \pi^{2} f \sigma^{2}\right)\right]\left(1-(\pi f \delta)^{2} / 2\right)} \\
& -(2 \pi f)\left((\pi f \delta)-(\pi f \delta)^{3} / 6\right) \pi \delta=0
\end{aligned}
$$

Expand and let $z=(\pi f)^{2}$, then

$$
\delta^{2}\left[12 \sigma^{2}+\delta^{2}\right] z^{2}-6\left[3 \delta^{2}+4 \sigma^{2}\right] z+24=0
$$

Which is a second degree equation, while

$$
\Delta=12\left[19 \delta^{4}-24 \delta^{2} \sigma^{2}+48 \sigma^{4}\right]
$$

Therefore,

$$
z=\frac{6\left[3 \delta^{2}+4 \sigma^{2}\right] \pm \sqrt{12\left[19 \delta^{4}-24 \delta^{2} \sigma^{2}+48 \sigma^{4}\right]}}{2 \delta^{2}\left[12 \sigma^{2}+\delta^{2}\right]}
$$


Hence, to reach a peak frequency value within the [3.1-10.6] GHz band, the expression of $f_{\text {peak }}$ is as provided in equation (25).

\section{Appendix B}

The frequency derivative of $S_{5,6}(f)$ provided in (31) is

$$
\begin{gathered}
S_{5,6}^{\prime}(f)=(2 \pi f)^{10} e^{-4(\pi f \sigma)^{2}} A_{5}{ }^{2} \times \\
{\left[-4 a_{5} a_{6} \pi \sqrt{\frac{2}{11}} \sigma(\sin (2 \pi f \delta)+2 \pi f \delta \cos (2 \pi f \delta))+8 \pi^{2} f a_{6}{ }^{2} \frac{2}{11} \sigma^{2}\right]} \\
+A_{5}{ }^{2}\left[20 \pi(2 \pi f)^{9} e^{-4(\pi f \sigma)^{2}}+(2 \pi f)^{10} e^{-4(\pi f \sigma)^{2}}\left(-8 \pi^{2} f \sigma^{2}\right)\right] \\
\times\left[a_{5}{ }^{2}-4 a_{5} \sin (2 \pi f \delta) a_{6} \sqrt{\frac{2}{11}} \sigma \pi f+a_{6}{ }^{2} \frac{2}{11} \sigma^{2}(2 \pi f)^{2}\right]
\end{gathered}
$$

At the peak frequency, $S_{5,6}^{\prime}(f)=0$, and knowing that $A_{5} \neq 0$, then for finite nonzero frequencies:

$$
\begin{gathered}
-4 a_{5} a_{6} \pi \sqrt{\frac{2}{11}} \sigma\left(\sin (2 \pi f \delta) 2 \pi f+2 f^{2} \pi \cos (2 \pi f \delta) 2 \pi \delta\right) \\
+16 \pi^{3} f^{2} a_{6}{ }^{2} \frac{2}{11} \sigma^{2}+\left(20 \pi-16 \pi^{3} f^{2} \sigma^{2}\right) \times \\
\left(a_{5}{ }^{2}-4 a_{5} \sin (2 \pi f \delta) a_{6} \sqrt{\frac{2}{11}} \sigma \pi f+a_{6}{ }^{2} \frac{2}{11} \sigma^{2}(2 \pi f)^{2}\right)=0
\end{gathered}
$$

What determines the pulse design is the relative amplitude of candidate waveforms, hence $a_{5}$ can be fixed at 1 for simplicity, while the resultant pulse dynamics will depend on the value of $a_{6}$ with respect to $a_{5}=1$. Therefore

$$
\begin{gathered}
-a_{6} \sqrt{\frac{2}{11}} \sigma\left(\sin (2 \pi f \delta) 2 \pi f+4 f^{2} \pi^{2} \cos (2 \pi f \delta) \delta\right) \\
+4 \pi^{2} f^{2} a_{6}{ }^{2} \frac{2}{11} \sigma^{2}+\left(5-4 \pi^{2} f^{2} \sigma^{2}\right) \times \\
\left(1-4 \sin (2 \pi f \delta) a_{6} \sqrt{\frac{2}{11}} \sigma \pi f+a_{6}{ }^{2} \frac{2}{11} \sigma^{2} 4 \pi^{2} f^{2}\right)=0
\end{gathered}
$$

While in appendix A, the third order Taylor series expansion of the sinusoids yielded an acceptable level of accuracy, a fourth order expansion is needed in this appendix for an improved accuracy:

$\sin (2 \pi f \delta)=(2 \pi f \delta)-(2 \pi f \delta)^{3} / 6$

$\cos (2 \pi f \delta)=1-(2 \pi f \delta)^{2} / 2+(2 \pi f \delta)^{4} / 24$ 
Following these approximations, equation (53) can be simplified to eventually reach a polynomial form:

$$
\begin{array}{r}
-2 a_{6} \sqrt{\frac{2}{11}} \sigma \delta(2 \pi f)^{2}+4 a_{6} \sqrt{\frac{2}{11}} \sigma(2 \pi f)^{4} \delta^{3} / 6-a_{6} \sqrt{\frac{2}{11}} \sigma(2 \pi f)^{6} \delta^{5} / 24 \\
+(2 \pi f)^{2} a_{6}{ }^{2} \frac{2}{11} \sigma^{2}+5+5\left(-2 a_{6} \sqrt{\frac{2}{11}} \sigma \delta+a_{6}{ }^{2} \frac{2}{11} \sigma^{2}\right)(2 \pi f)^{2} \\
+5 \times 2 a_{6} \sqrt{\frac{2}{11}} \sigma \delta^{3}(2 \pi f)^{4} / 6-(2 \pi f)^{2} \sigma^{2} \\
-\sigma^{2}\left(-2 a_{6} \sqrt{\frac{2}{11}} \sigma \delta\right. \\
\left.+a_{6}{ }^{2} \frac{2}{11} \sigma^{2}\right)(2 \pi f)^{4}-2 \sigma^{2} a_{6} \sqrt{\frac{2}{11}} \sigma \delta^{3}(2 \pi f)^{6} / 6=0
\end{array}
$$

Let $U=(2 \pi f)^{2}$, then arrange to obtain:

$$
\begin{gathered}
{\left[-12 a_{6} \sqrt{\frac{2}{11}} \sigma \delta+6 a_{6}{ }^{2} \frac{2}{11} \sigma^{2}-\sigma^{2}\right] U} \\
+\sqrt{\frac{2}{11}} a_{6} \sigma\left[14 \delta^{3} / 6+2 \sigma^{2} \delta-a_{6} \sqrt{\frac{2}{11}} \sigma^{3}\right] U^{2} \\
+a_{6} \sqrt{\frac{2}{11}} \delta^{3} \sigma\left[-\delta^{2} / 24-\sigma^{2} / 3\right] U^{3}+5=0
\end{gathered}
$$

\section{References}

[1] Porcino, D., \& Hirt, W. (2003). Ultra-wideband radio technology: potential and challenges ahead. IEEE communications magazine, 41(7), 66-74.

[2] M. Ghavami, L. Michael, and R. Kohno, "Ultra wideband signals and systems in communication engineering," John Wiley \& Sons, 2007.

[3] Ali, R., Liu, R., Nayyar, A., Qureshi, B., \& Cao, Z. (2021). Tightly Coupling Fusion of UWB Ranging and IMU Pedestrian Dead Reckoning for Indoor Localization. IEEE Access.

[4] G. R. Aiello and G. D. Rogerson, "Ultra-wideband wireless systems," IEEE Microwave Magazine, 4(2), pp. 36-47, 2003.

[5] Feghhi, R., Oloumi, D., \& Rambabu, K. (2020). Tunable Subnanosecond Gaussian Pulse Radar Transmitter: Theory and Analysis. IEEE Transactions on Microwave Theory and Techniques, 68(9), 3823-3833.

[6] Fontana, R. J. (2004). Recent system applications of short-pulse ultrawideband (UWB) technology. IEEE Transactions on microwave theory and techniques, 52(9), 2087-2104. 
[7] Chandel, R., Gautam, A. K., \& Rambabu, K. (2018). Tapered fed compact UWB MIMO-diversity antenna with dual band-notched characteristics. IEEE Transactions on Antennas and Propagation, 66(4), 1677-1684.

[8] Nazeri, A. H., Falahati, A., \& Edwards, R. M. (2019). A novel compact fractal UWB antenna with triple reconfigurable notch reject bands applications. AEU-International Journal of Electronics and Communications, $101,1-8$.

[9] Yu, K., Wen, K., Li, Y., Zhang, S., \& Zhang, K. (2018). A novel NLOS mitigation algorithm for UWB localization in harsh indoor environments. IEEE Transactions on Vehicular Technology, 68(1), 686-699.

[10] I. Oppermann, M. Hämäläinen, and J. Iinatti, "UWB: theory and applications," John Wiley \& Sons, 2005.

[11] Taki, H., Azou, S., Hamie, A., Morel, P., Al Housseini, A., Alaeddine, A., \& Sharaiha, A. (2018). Chirping techniques for enhancing the performance of SOA-based UWB over fiber systems: An experimental demonstration. AEU-International Journal of Electronics and Communications, 94, 221225 .

[12] Federal Communications Commission, "Revision of Part 15 of the commission's rules regarding ultra-wideband transmission systems, FIRST REPORT AND ORDER," ET Docket 98-153, FCC 02-48, pp. 1-118, February 14, 2002.

[13] Hamalainen, M., Hovinen, V., Tesi, R., Iinatti, J. H., \& Latva-aho, M. (2002). On the UWB system coexistence with GSM900, UMTS/WCDMA, and GPS. IEEE Journal on Selected areas in Communications, 20(9), $1712-1721$.

[14] Niemela V, Haapola J, Hamalainen M, Iinatti J. An Ultra Wideband Survey: Global Regulations and Impulse Radio Research Based on Standards. IEEE Communications Surveys \& Tutorials. 2017 May 19.

[15] Di Benedetto, M. G. (Ed.). (2006). UWB communication systems: a comprehensive overview.

[16] M. Z. Win and R. A. Scholtz, "Impulse radio: How it works," IEEE Communications Lett., 2(2), pp. 36-38, 1998.

[17] J. R. Fernandes and D. Wentzloff, "Recent advances in IR-UWB transceivers: An overview," In Circuits and Systems (ISCAS), Proceedings of 2010 IEEE International Symposium, pp. 3284-3287, 2010. 
[18] Lee, G., Park, J., Jang, J., Jung, T., \& Kim, T. W. (2019). An IR-UWB CMOS Transceiver for High-Data-Rate, Low-Power, and Short-Range Communication. IEEE Journal of Solid-State Circuits.

[19] Liang, X., Zhang, H., Ye, S., Fang, G., \& Gulliver, T. A. (2018). Improved denoising method for through-wall vital sign detection using UWB impulse radar. Digital Signal Processing, 74, 72-93.

[20] Shehata, M., Said, M. S., \& Mostafa, H. (2019). A generalized framework for the performance evaluation of microwave photonic assisted ir-uwb waveform generators. IEEE Systems Journal, 13(4), 3724-3734.

[21] Du, C., Liu, F., Li, X., Zhang, Z., \& Dong, W. (2020). UWB quadruplet signal generation based on a DP-QPSK modulator and a delay-line filter. Applied optics, 59(14), 4404-4409.

[22] Kaya, E., \& Entesari, K. (2020, January). A Broadband CMOS Pulse Generator for UWB Systems. In 2020 IEEE Radio and Wireless Symposium (RWS) (pp. 9-11). IEEE.

[23] Biswas, D. K., \& Mahbub, I. (2021, January). A Low-Power Duty-Cycled Impulse-Radio Ultrawideband (IR-UWB) Transmitter with Bandwidth and Frequency Reconfigurability Scheme Designed in $180 \mathrm{~nm}$ CMOS Process. In 2021 IEEE Radio and Wireless Symposium (RWS) (pp. 49-52). IEEE.

[24] Mu, H., Wang, M., Tang, Y., Zhang, J., \& Jian, S. (2018). Photonic generation of FCC-compliant UWB pulses based on modified Gaussian quadruplet and incoherent wavelength-to-time conversion. Optics Communications, 411, 170-174.

[25] Mu, H., Wang, M., \& Li, M. (2019). Power-efficient FCC-compliant UWB generator using polarization-maintaining FBG-based spectral shaper and incoherent wavelength-to-time mapping. Optical Fiber Technology, 50, 271-276.

[26] Kikkawa, T., Masui, Y., Toya, A., Ito, H., Hirano, T., Maeda, T., ... \& Iwata, A. (2020). CMOS Gaussian Monocycle Pulse Transceiver for Radar-Based Microwave Imaging. IEEE Transactions on Biomedical Circuits and Systems, 14(6), 1333-1345.

[27] Phan, T. A., Krizhanovskii, V., Han, S. K., Lee, S. G., Oh, H. S., \& Kim, N. S. (2007, May). 4.7 pJ/pulse 7 th derivative Gaussian pulse generator for Impulse Radio UWB. In 2007 IEEE International Symposium on Circuits and Systems (pp. 3043-3046). IEEE. 
[28] Vauche, R., Muhr, E., Fourquin, O., Bourdel, S., Gaubert, J., Dehaese, N., ... \& Ouvry, L. (2017). A 100 MHz PRF IR-UWB CMOS transceiver with pulse shaping capabilities and peak voltage detector. IEEE Transactions on Circuits and Systems I: Regular Papers, 64(6), 1612-1625.

[29] Nikookar, H., \& Prasad, R. (2008). Introduction to ultra wideband for wireless communications. Springer Science \& Business Media.

[30] Kim, H., \& Joo, Y. (2005, June). Fifth-derivative Gaussian pulse generator for UWB system. In 2005 IEEE Radio Frequency integrated Circuits (RFIC) Symposium-Digest of Papers (pp. 671-674). IEEE.

[31] Hu, B., \& Beaulieu, N. C. (2005). Pulse shapes for ultrawideband communication systems. IEEE Transactions on wireless communications, 4(4), 1789-1797.

[32] Sheng, H., Orlik, P., Haimovich, A. M., Cimini, L. J., \& Zhang, J. (2003, May). On the spectral and power requirements for ultra-wideband transmission. In IEEE International Conference on Communications, 2003. ICC'03. (Vol. 1, pp. 738-742). IEEE.

[33] Taki, H., Azou, S., Hamie, A., Al Housseini, A., Alaeddine, A., \& Sharaiha, A. (2016). Improving the power efficiency of SOA-based UWB over fiber systems via pulse shape randomization. Optical Fiber Technology, 31, 161-167.

[34] Menon, M. D., Rodrigues, J., \& Gudino, L. J. (2020, July). Synthesis of UWB Pulse Shaper for Efficient Pulse Propagation in Human Tissue. In 2020 12th International Symposium on Communication Systems, Networks and Digital Signal Processing (CSNDSP) (pp. 1-5). IEEE.

[35] Rahman, M., Haider, A., \& Naghshvarianjahromi, M. (2020). A systematic methodology for the time-domain ringing reduction in UWB bandnotched antennas. IEEE Antennas and Wireless Propagation Letters, 19(3), 482-486.

[36] Nowakowski, M., \& Idzkowski, A. (2020, July). Ultra-wideband signal transmission according to European regulations and typical pulses. In 2020 International Conference Mechatronic Systems and Materials (MSM) (pp. 1-4). IEEE.

[37] Abraha, S. T., Okonkwo, C., Gamage, P. A., Tangdiongga, E., \& Koonen, T. (2012). Routing of power efficient IR-UWB wireless and wired services for in-building network applications. Journal of Lightwave Technology, 30(11), 1651-1663. 
[38] Taki, H., Azou, S., Hamie, A., Al Housseini, A., Alaeddine, A., \& Sharaiha, A. (2017). Simple pre-distortion schemes for improving the power efficiency of SOA-based IR-UWB over fiber systems. Optics communications, 382, 225-231.

[39] H. Taki, S. Azou, A. Hamie, A. Al Housseini, A. Alaeddine, and A. Sharaiha, "On phaser-based processing of impulse radio UWB over fiber systems employing SOA," Optical Fiber Technology, 36, 33-40, 2017.

[40] Radic, J., Brkic, M., Djugova, A., Videnovic-Misic, M., Goll, B., \& Zimmermann, H. (2020). Ultra-low power low-complexity 3-7.5 GHz IR-UWB transmitter with spectrum tunability. IET Circuits, Devices \& Systems, 14(4), 521-527.

[41] de Jesus Aragão, A., de Carvalho, D., Sanches, B., \& Van Noije, W. A. (2020, February). An improved confocal algorithm for breast cancer detection using UWB signals. In 2020 IEEE 11th Latin American Symposium on Circuits \& Systems (LASCAS) (pp. 1-4). IEEE.

[42] Taki, H., Mansour, A., Azou, S., Nasser, A., \& Yao, K. (2020). Pulse parity modulation for impulse radio UWB transmission based on non-coherent detection. Physical Communication, 40, 101061.

[43] Abraha, S. T., Okonkwo, C., Yang, H., Visani, D., Shi, Y., Jung, H. D., ... \& Koonen, T. (2011). Performance evaluation of IR-UWB in short-range fiber communication using linear combination of monocycles. Journal of lightwave technology, 29(8), 1143-1151.

[44] Mirshafiei, M., Abtahi, M., \& Rusch, L. A. (2012). Ultra-wideband pulse shaping: bypassing the inherent limitations of the Gaussian monocycle. IET communications, 6(9), 1068-1074.

[45] Peilin, L., Qingsong, Z., Jianyun, Z., \& Lei, L. (2018, July). Pulse Design for UWB Based on the Combination of Gaussian Derivatives. In 2018 10th International Conference on Communication Software and Networks (ICCSN) (pp. 438-441). IEEE.

[46] Maaref, A., \& Elahmar, S. A. (2021). UWB Pulse Design Method Using Firefly Algorithm. Radioelectronics and Communications Systems, 64(3), 140-146.

[47] Rodrigues, J., MK, D. M., Lonappan, L., \& Gudino, L. J. (2020). Spectral Efficient Pulse Shaping for Impulse Radio Ultra Wideband Communications. Helix, 10(02), 226-231.

[48] Amini, A., Esfahani, P. M., Ghavami, M., \& Marvasti, F. (2019). UWB orthogonal pulse design using Sturm-Liouville boundary value problem. 
Signal Processing, 159, 147-158.

[49] J. E. Mitchell, P. P. Pardalos, and M. G. C. Resende. Interior point methods for combinatorial optimization. In D.-Z. Du and P. Pardalos, editors, Handbook of Combinatorial Optimization , vol. 1, pp. 189-297. Kluwer Academic Publishers, 1998.

[50] Byrd, R. H., Hribar, M. E., \& Nocedal, J. (1999). An interior point algorithm for large-scale nonlinear programming. SIAM Journal on Optimization, 9(4), 877-900.

[51] Wachter, A., \& Biegler, L. T. (2006). On the implementation of an interior-point filter line-search algorithm for large-scale nonlinear programming. Mathematical programming, 106(1), 25-57.

[52] Kim, S. J., Koh, K., Lustig, M., Boyd, S., \& Gorinevsky, D. (2007). An Interior-Point Method for Large-Scale 11-Regularized Least Squares. IEEE journal of selected topics in signal processing, 1(4), 606-617.

[53] Khalesi, H., Ghods, V. An Optimized IR-UWB Communication System with Interference Reduction on a Narrowband System Using Genetic Algorithm. Wireless Pers Commun (2021).

[54] Han, S. H., \& Lee, J. H. (2005). An overview of peak-to-average power ratio reduction techniques for multicarrier transmission. IEEE wireless communications, 12(2), 56-65.

[55] Niu, W., Chen, H., Zhang, J., Ha, Y., Zou, P., \& Chi, N. (2021). Nonlinearity Mitigation based on Modulus Pruned Look-up Table for Multi-bit Delta-sigma 32-CAP Modulation in Underwater Visible Light Communication System. IEEE Photonics Journal.

[56] Liu, K., \& Liu, Y. A. (2021). Adjustable Nonlinear Companding Transform Based on Scaling of Probability Density Function for PAPR Reduction in OFDM Systems. IEEE Transactions on Broadcasting.

[57] Witrisal K, Leus G, Janssen GJ, Pausini M, Trösch F, Zasowski T, Romme J. Noncoherent ultra-wideband systems. IEEE Signal Processing Magazine. 2009 Jul;26(4).

[58] J.E. Padgett, J.C. Koshy, A.A. Triolo, Physical-layer modeling of UWB interference effects, Telcordia Technologies Report to DARPA (NETEX Program), 2003.

[59] Pearce, A., Nie, H., \& Chen, Z. (2012, June). Spectral spike reduction for ultra-wideband impulse radio system. In 2012 IEEE/MTT-S International Microwave Symposium Digest (pp. 1-3). IEEE. 
[60] Chao, Y. L., \& Scholtz, R. A. (2005). Ultra-wideband transmitted reference systems. IEEE Transactions on Vehicular Technology, 54(5), 1556-1569.

\section{Statements and Declarations}

On behalf of all authors, the corresponding author states that there is no conflict of interest.

The authors also declare that no funds, grants, or other support were received during the preparation of this manuscript.

All authors contributed to the study conception and design.

Haidar Taki: Material preparation, Investigation, Methodology, Software, and analysis. Chadi Abou-Rjeily: Conceptualization, Review, and editing. 